Bull. Iraq nat. Hist. Mus.

\title{
SURVEY OF INSECTS IN SOME SOUTHERN IRAQI MARSHES
}

\author{
Hanaa H. Al-Saffar and Razzaq Shalan Augul \\ Iraq Natural History Research Center and Museum, University of Baghdad, Baghdad, Iraq. \\ •Corresponding author email: dr.hanaa66@nhm.uobaghdad.edu.iq \\ Received Date: 13 Sept. 2021, Accepted Date: 28 Nov. 2021, Published Date: 20 December 2021 \\ (c) (i) \\ This work is licensed under a Creative Commons Attribution 4.0 International License
}

\section{ABSTRACT}

This study included a survey and review of the scientific names of the marsh insects (aquatic and surrounding it) for the purpose of unifying and updating the database.

The survey reveals 109 species under 77 genera that belong to 32 families and 7 orders as follow: Coleoptera (44 species), Diptera (7 species) Ephemeroptera (2 species), Hemiptera (14 species), Hymenoptera (11 species), Lepidoptera (2 species) and Odonata with 29 species.

Information of specimens' collection for each species, synonyms and geographical distribution were provided.

Key words: Insects, Iraq, Marshes, Survey, Synonyms.

\section{INTRODUCTION}

The Iraqi marshlands cover an area of 15000-20000 sq. km in the lower part of the Mesopotamian basin where the Tigris and Euphrates Rivers flow; that lie on a softly sloping plan which causes the two rivers to meander which branches form the marshes. The marshes lay on the heavy fluvial sediments that are carried by the rivers in the area (Al-Ansari et al., 2012). The marshes of Iraq are one of the largest water area and with completely ecosystem in the Middle East. They are situated at the southern part among Basra, Maysan and Thi Qar Provinces. The marshes consist of three main regions: Central Marshes, Hawizeh and AlHammar Marsh (Bedair et al., 2006).

According to the previous field studies, the marshes including a wide range of diversity with their levels which appeared with high fluctuations; on the other hand, the marshes status was affected in high degree by high flood and drying seasons (Al-Hili, 1977; Richardson et al., 2005). In the marshes of southern Iraq, the pervious investigation is focused on the water quality, zooplankton and phytoplankton diversity, macro-invertebrates and birds; however, less attention was made toward micro-invertebrates, insects, and herpetofauna (Iraqi Ministry of Health and Environment, 2017). 
From the other side, there were eleven water beetles species of the family Haliplidae collected from the Shatt Al Arab and marshes of southern Iraq (Ali, 1976); also lists 55 species of dytiscid water beetles (Family, Dytiscidae) and fifteen species of gyrinid beetles (Family, Gyrinidae) (Ali, 1978 a, b). As well, Twenty five dragonfly species are known to occur in the central and southern Iraqi Marshlands (Boudot et al., 2009). Hassan et al. (2000) reported Anax spp. and Ischnura evansi Morton, 1919 (Odonata, Coenagrionidae) from several stations along Shatt al-Arab; Ali et al. (2002) studied the seasonal abundances of $I$. evansi and Brachythemis fuscopalliata Selys, 1887 (Odonata, Libellulidae) in the Qarmat Ali region near Basrah. Dragonflies inhabiting rivers and marshes in arid regions such as southern Iraq, such as Hemianax ephippiger and Ischnura evansi are tolerant of high salinity (Corbet, 1999).

Generally, the biodiversity in the re-inundated southern marshes of Iraq has attracted the attention of many authors; however, still little is known about certain biota the marshes such as fungi, invertebrates such as annelids and aquatic insects, and wild mammals. The biodiversity in the Iraqi marshes is considered low when it compared with other wetlands in the world (Hussain, 2014).

Updating checklists in this ecosystem still out of date; therefore, the study aimed to prepare an updating checklist of the insect species that live in marshes or closely to this unique ecosystem.

\section{MATERIALS AND METHODS}

The specimens were collected from different localities Southern Marshes of Iraq from three provinces (Basra, Maysan and Thi Qar) by using air and water nets and sometimes with hands. The adult insects were killed by freezing for 24 hours while the larvae killed by alcohol 70\%. The localities and date of collection were recorded. The specimens were classified and identified to orders, families, genera and species by using different classification keys such as: Coe et al. (1950); Cranston and Judd (1989); Askew (2004); Nieser (2004); Dijkstra and Lewington (2006); Kalkman, (2006); Madden (2010); Kumar (2012); Perveen et al. (2014); Al-Hashmi et al. (2018); Novoselsky et al. (2018) and Alhejoj et al. (2020).

The diagnosis of insects by the authors and the specimens were deposed at the Department of Entomology and Invertebrates, Iraq Natural History Research Center and MuseumUniversity of Baghdad. The synonyms of species have been verified according to GBIF Secretariat (2021).

\section{RESULTS AND DISCUSSION}

In this investigation showed 110 species at southern marshes belonging to seven orders: Coleoptera (45), Diptera (7), Ephemeroptera (2); Hemiptera (15); Hymenoptera (11); Lepidoptera (2) and Odonata with 29 species, as below: 


\section{(A) Order, Coleoptera}

(1) Family, Dytiscidae

Genus, Agabus Leach, 1817

Synonyms: Acathodes Seidlitz, 1887

Acatodes Thomson, 1859

Agabinectes Guignot, 1933

Allonychus Zaitzev, 1905

Apator Semenov, 1898

Arctodytes Thomson, 1874

Badynectus Seidlitz, 1872

Carrhydrus Fall, 1922

Dichodytes Thomson, 1886

Dichonectes Guignot, 1945

Eriglenus Thomson, 1860

Gabinectes Guignot, 1931

Gaurodytes Thomson, 1859

Heteronychus Seidlitz, 1887

Mesogabus Gueorguiev, 1969

Metronectes Sharp, 1882

Necticus Hope, 1838

Neonecticus Guignot, 1951

Scytodytes Seidlitz, 1887

Xanthodytes Seidlitz, 1887

Agabus biguttatus (Olivier, 1795)

Synonym: Dytiscus biguttatus Olivier, 1795

Material examined: 7 specimens; Maysan Province, Hawizeh Marshes, Umm An-Ni'aaj, 19.vii.2020

Distribution: Iraq (Abdul Karim, 1978); Jordon, Saudi Arabia and Yemen (Ramadan and Ramadan, 2021).

Agabus caraboides Sharp, 1882

Material examined: 1 specimen; Maysan Province, Hawizeh Marshes, Umm An-Ni'aaj, 19.vii. 2020

Distribution: Iraq (Zimmermann, 1920); Turkey and Greece (GBIF Secretariat, 2021).

Agabus conspersus (Marsham, 1802)

Synonyms: Agabus corsicus Guignot, 1932

Dytiscus conspersus Marsham, 1802

Material examined: 5 specimens; Maysan Province, Hawizeh Marshes, Umm An-Ni'aaj, 19.vii.2020

Distribution: Iraq (Zimmermann, 1920; Nilson, 2003); Jordon and Kuwait (Ramadan and Ramadan, 2021). 
Agabus guttatus (Paykull, 1798)

Synonym: Dytiscus guttatus Paykull, 1798

Material examined: 4 specimens, Maysan Province, Hawizeh Marshes, Umm An-Ni'aaj, 19.vii.2020.

Distribution: Iraq (Abdul-Karim, 1978); Turkey (Darilmaz and kayak, 2009).

Agabus paludosus (Fabricius, 1801)

Synonym: Dytiscus paludosus Fabricius, 1801

Material examined: 3 specimens, Maysan Province: Hawizeh Marshes, Umm An-Ni'aaj, 30.vi.2020

Distribution: Iraq (Abdulhasan et al., 2009); Turkey (Darilmaz and kayak, 2009).

Agabus safei Abdul-Karim \& Ali, 1986

Material examined: 5 specimens, Thi- Qar Province, Al-Chibayish Marshes, 3.vii.2020.

Distribution: Iraq (Abdul-Karim and Ali, 1986).

Genus, Bidessus Sharp, 1882

Synonym: Callioprus Adam, 1996

Bidessus exorantus (Reich and Saulcy, 1855)

Synonym: Hydroporus exorantus Reich and Saulcy, 1855

Material examined: 2 specimens; Thi- Qar Province, Al-Chibayish Marshes, 3.vii.2020.

Distribution: Iraq (Abdul-Karim, 1978); Turkey (Darilmaz and kayak, 2009).

Genus, Colymbetes Clairville, 1806

Synonyms: Cymatopterus Dejean, 1833

Lymnaeus Gistel, 1834

Colymbetes fuscus (Linnaeus, 1758)

Synonym: Dytiscus fuscus Linnaeus, 1758

Material examined: 1 specimen; Thi-Qar Province, Al-Chibayish Marshes, 30.iv.2020.

Distribution: Iraq (Abdul-Karim, 1978); Liberian Peninsula (Garrido and Munilla, 2008);

Turkey (Darilmaz and kayak, 2009); Croatia (Turić et al., 2011); Iran (Taher and Heydarnejad, 2019).

Colymbetes piceus Klug, 1834

Material examined: 3 specimens; Thi-Qar Province, Al-Chibayish Marshes, 30.iv.2020.

Distribution: Iraq (Derwesh, 1965); Kuwait, Saudi Arabia (Ramadan and Ramadan, 2021).

Genus, Copelatus Erichson, 1832

Copelatus pulchellus (Klug, 1834)

Synonym: Copelatus mimetes Guignot, 1957 


\section{Al-Saffar and Augul}

Material examined: 13 specimens; Thi-Qar Province, Al-Chibayish Marshes, 30.iv.2020. Distribution: Iraq (Abdul-Karim, 1978); widespread to Western and Eastern Africa (Perissinotto et al., 2016).

Genus, Cybister Curtis, 1827

Synonyms: Alocomerus Brinck, 1945

Cybisteter Bedel, 1880

Gschwendtnerhydrus Brinck, 1945

Megadytoides Brinck, 1945

Meganectes Brinck, 1945

Melanectes Brinck, 1945

Nealocomerus Brinck, 1945

Neocybister Miller, Bergsten \& Whiting, 2007

Scaphinectes Ádám, 1993

Trochalus Dejean, 1833

Trogulus Brodie, 1874

Trogus Leach, 1817

\section{Cybister tripunctatus (Olivier, 1795)}

Synonyms: Cybister szechwanensis Falkenström, 1936

Dytiscus tripunctatus Olivier, 1795

Material examined: 11 specimens; 3 specimens, Maysan Province, Hawizeh Marshes, Umm An-Ni'aaj, 19.vii. 2020; 8 specimens, Thi- Qar Province, Al-Chibayish Marshes, 30.iv.2020.

Distribution: Iraq (Abdul-Rassoul,1976); Taiwan(Nilsson et al., 1995); Kuwait (AlHouty, 2004); Turkey (Darilmaz and kayak, 2009); West Africa (Yapo et al., 2013).

Genus, Eretes Laporte, 1833

Synonyms: Eunectes Erichson, 1832

Hogrus Zubkov, 1837

Nogrus Dejean, 1833

Eretes sticticus (Linnaeus, 1767)

Synonyms: Dytiscus sticticus Linnaeus, 1767

Eretes occidentalis (Erichson, 1847)

Eretes stricticus (Linnaeus, 1767)

Material examined: 3 specimens, Maysan Province, Hawizeh Marshes, Umm An-Ni'aaj, 19.vii.2020.

Distribution: Iraq (Abdul-Karim, 1978); Taiwan (Nilsson et al., 1995); Southern of United States and Peru (Miller, 2002); Iran, Pakistan (Hájek, 2006); Egypt (Younes, 2008); Kuwait (Al-Houty, 2009); Turkey (Darilmaz and kayak, 2009); Algeria, Botswana, Ethiopia, Tunisia, Sudan, Morocco, Zimbabwe, South Africa, Namibia, Madagascar, Tanzania, Kenya, Senegal, Mali, India, Korea, Guam, Uzbekistan, Turkmenistan, Japan, Thailand, Philippines, Vietnam, Ecuador, Mexico, Portugal, Spain, Greece, Italy and Australia (GBIF Secretariat, 2021). 
Genus, Herophydrus Sharp, 1880

Synonym: Dryephorus Guignot, 1950

Herophydrus guineensis (Aubé, 1838)

Synonyms: Herophydrus mutatus (Gemminger \& Harold, 1868)

Hygrotus guineensis (Aubé, 1838)

Hyphydrus guineensis Aubé, 1838

Material examined: 4 specimens; 3 specimens, Maysan Province, Hawizeh Marshes, Umm An-Ni'aaj, 19.vii.2020; 1 specimen, Thi- Qar Province, Al-Chibayish Marshes, 30.iv.2020.

Distribution: Iraq (Abdul-Karim, 1978); Turkey (Darilmaz and kayak, 2009); Egypt (Salah and Cueto, 2014).

Herophydrus musicus (Klug, 1834)

Synonyms: Coelambus interruptus Sharp, 1882

Hydroporus fractilinea Solsky, 1874

Hydroporus musicus Klug, 1834

Hygrotus alei Abdul-Karim \& Ali, 1986

Material examined: 5 specimens; Thi-Qar Province, Al-Chibayish Marshes, 30.iv.2020.

Distribution: In Iraq, previously recorded as Hygrotus alei (Abdul-Karim and Ali, 1986); Iran, Pakistan (Hájek, 2006); Turkey (Darilmaz and kayak, 2009); Kuwait (Al-Houty, 2009); Egypt (Salah and Cueto, 2014).

Genus, Hydaticus Leach, 1817

Synonyms: Guignotites Brinck, 1943

Hydaticinus Guignot, 1950

Icmaleus Gistel, 1856

Isonotus Guignot, 1936

Pleurodytes Régimbart, 1899

Prodaticus Sharp, 1882

Hydaticus dorsiger Aubé, 1838

Material examined: 5 specimens, Thi- Qar Province, Al-Chibayish Marshes, 3.vii.2020. Distribution: Iraq (Abdul-Karim, 1978); Widely distributed species, (Guignot, 1961).

Hydaticus ponticus Sharp, 1882

Material examined: 1 specimen, Thi- Qar Province, Al-Chibayish Marshes, 30.iv.2020. Distribution: Iraq (Abdul-Karim, 1978); Iran, Pakistan (Hájek, 2006); Turkey (Darilmaz and kayak, 2009).

Genus, Hydroglyphus Motschulsky, 1853

Synonym: Guignotus Houlbert, 1934

Hydroglyphus confusus (Klug, 1834) 


\section{Al-Saffar and Augul}

Synonym: Hydroporus confusus Klug, 1834

Material examined: 6 specimens, Maysan Province: Hawizeh Marshes, Umm An-Ni'aaj, 30.vi.2020

Distribution: Iraq (Abdul-Karim, 1978); Turkey (Darilmaz and kayak, 2009); Egypt (Salah and Cueto, 2014); Croatia (Turić et al., 2017).

Genus, Hydroporus Clairville, 1806

Synonym: Hydrotarsus Falkenström, 1938

Hydroporus tessellatus (Drapiez, 1819)

Synonym: Dytiscus tessellatus Drapiez, 1819

Distribution: Iraq (Abdul-Karim, 1978); Liberian Peninsula (Garrido and Munilla, 2008);

Turkey (Darilmaz and Kayak, 2009); Egypt (Salah and Cueto, 2014).

Genus, Hydrovatus Motschulsky, 1853

Synonyms: Hydatonychus Kolbe, 1883

Oxynoptilus Schaum, 1868

Pseudhydrovatus Peschet, 1924

Vathydrus Guignot, 1954

Hydrovatus badeni Sharp, 1882

Material examined: 3 specimens, Thi- Qar Province, Al-Chibayish Marshes, 3.vii.2020.

Distribution: Iraq (Abdul-Karim, 1978).

Hydrovatus clypealis Sharp, 1876

Synonym: Oxynoptilus clypealis (Sharp, 1876)

Material examined: 1 specimen, Thi- Qar Province, Al-Chibayish Marshes, 29.viii.2020.

Distribution: Iraq (Abdul-Karim, 1978); Egypt (Salah and Cueto, 2014).

Hydrovatus meridionalis Abdul-Karim \& Ali, 1986

Material examined: 3 specimens, Maysan Province, Hawizeh Marshes, Umm An-Ni'aaj, 19.vii.2020

Distribution: Iraq (Abdul-Karim and Ali, 1986)

Genus, Hygrotus Stephens, 1828

Synonyms: Drryephorus Guignot, 1950

Heroceras Guignot, 1950

Herophydrus Sharp, 188

Hyphoporus Sharp, 1880

\section{Hygrotus sp.}

Material examined: 1 specimen, Maysan Province, Hawizeh Marshes, Umm An-Ni'aaj, 19.vii.2020.

Distribution: Iraq (Abdulhasan et al., 2009) 
Genus, Hyphoporus Sharp, 1880

Hyphoporus solieri (Aubé, 1838)

Synonym: Agabus solieri Aubé, 1837

Material examined: 7 specimens, Maysan Province: Hawizeh Marshes, Umm An-Ni'aaj, 29.viii. 2020

Distribution: Iraq (Abdul-Karim, 1978); Egypt (Salah and Cueto, 2014); Kuwait (Amr, 2021)

Genus, Hyphydrus Illiger, 1802

Synonyms: Actobaena Gistel, 1856

Allophydrus Zimmermann, 1930

Apriophorus Guignot, 1938

Aulacodytes Guignot, 1938

Hyphidrus Illiger, 1802

Hyphidrus Sturm, 1826

Pachytes Montrouzier, 1860

Hyphydrus aubei Ganglbauer, 1891

Material examined: 5 specimens, Thi- Qar Province, Al-Chibayish Marshes, 3.vii.2021

Distribution: Iraq (Abdul-Karim, 1978); Liberian Peninsula (Garrido and Munilla, 2008).

Genus, Laccophilus Leach, 1815

Synonym: Lacophilus Sturm, 1826

Laccophilus hyalinus (De Geer, 1774)

Synonyms: Dyticus marmoratus Fourcroy, 1785

Dytiscus hyalinus De Geer, 1774

Laccophilus interruptus (Panzer, 1795)

Laccophilus testaceus Aubé, 1837

Material examined: 8 specimens, Thi- Qar Province, Al-Chibayish Marshes, 29.viii.2020.

Distribution: Iraq (Abdul-Karim, 1978); Turkey (Darilmaz and kayak, 2009); Iran

(Vafael et al., 2009); Kis- Balaton (Lökkös, 2014); Croatia (Turić et al., 2017 ); Iran

(Taher and Heydarnejad, 2019); Ireland (Nelson et al., 2019).

Laccophilus minutus (Linnaeus, 1758)

Synonyms: Dytiscus dinutus Linnaeus, 1758

Dytiscus minutus Linnaeus, 1758

Laccophilus obscurus (Panzer, 1795)

Laccophilus variolosus (Herbst, 1784)

Material examined: 2 specimens, Thi- Qar Province, Al-Chibayish Marshes, 3.vii.2021.

Distribution: Iraq (Abdul-Karim, 1978); Jordan (Ramadan and Ramadan, 2021).

Laccophilus poecilus Klug, 1834 
Al-Saffar and Augul

Synonyms: Dyticus variegatus Germar, 1812

Dytiscus variegatus Germar \& Kaulfuss, 1817

Laccophilus ponticus Sharp, 1882

Laccophilus variegatus (Germar, 1812)

Material examined: 3 specimens, Maysan Province: Hawizeh Marshes, Umm An-Ni'aaj, 29.viii. 2020

Distribution: Iraq (Abdul-Karim, 1978 as Laccophilus ponticus); Kuwait (Al-Houty, 2004); Turkey (Darilmaz and kayak, 2009); Croatia (Turić et al., 2017).

Laccophilus sharpi Régimbart, 1889

Material examined: 9 specimens, Maysan Province: Hawizeh Marshes, Umm An-Ni'aaj, 29.viii. 2020

Distribution: Iraq (Abdul-Karim, 1978); Iran, Pakistan (Hájek, 2006).

Genus, Nebrioporus Régimbart, 1906

Synonyms: Bistictus Guignot, 1942

Potamodytes Zimmermann, 1919

Potamonectes Zimmermann, 1921

Rhabdonectes Houlbert, 1934

Zimmermannius Guignot, 1942

Nebrioporus laeviventris (Reiche \& Saulcy, 1855)

Synonym: Hydroporus laeviventris Reiche \& Saulcy, 1855

Material examined :2 specimens, Maysan Province: Hawizeh Marshes, Umm An-Ni'aaj, 23.vi.2021.

Distribution: Iraq (as Stictotarsus laeviventris Reiche and Saulcy, 1855) Abdul-Karim, 1978); Taiwan (Nilsson et al., 1995); Turkey (Darilmaz and kayak, 2009).

Genus, Platambus Thomsom, 1859

Synonyms: Agraphis Guignot, 1954

Allogabus Guignot, 1954

Anagabus Jakovlev, 1897

Colymbinectes Falkenström, 1936

Neoplatynectes Vazirani, 1970

Paraplatynects Vazirani, 1970

Platambus maculatus (Linnaeus, 1758)

Synonyms: Agabus maculatus (Linnaeus, 1758)

Dyticus hebraicus Fourcroy, 1785

Dytiscus maculatus Linnaeus, 1758

Material examined :4 specimens, Maysan Province: Hawizeh Marshes, Umm An-Ni'aaj, 23.vi.2021.

Distribution: Iraq (Abdul-Karim, 1978); Turkey (Darilmaz and kayak, 2009); Mongollia (Enkhnasan and Blodgiv, 2019). 
Genus, Rhantus Dejean, 1833

Synonyms: Anisomera Brullé, 1834

Anisomeria Brinck, 1943

Ilybiomorphus Porta, 1923

Rantogiton Des Gozis, 1910

Rantus Dejean, 1833

Rhantus Agassiz, 1846

Senilites Brinck, 1948

Rhantus suturalis (MacLeay, 1825)

Synonyms: Colymbetes montrouzieri Lucas, 1860

Colymbetes pulverosus Stephens, 1828

Colymbetes suturalis W.S.MacLeay, 1825

Dyticus punctatus Fourcroy, 1785

Rhanthus suturalis (W.S.MacLeay, 1825)

Rhantus pulverosus (Stephens, 1828)

Material examined: 1 specimen, Thi- Qar Province, Al-Chibayish Marshes, 3.vii.2021

Distribution: Iraq (recorded as Rhantus pulverosus (Stephens, 1828) ,Abdul-Karim, 1978); Taiwan ; (Nilsson et al., 1995); Iran , Pakistan (Hájek, 2006); Liberian Peninsula (Garrido and Munilla, 2008); Turkey (Darilmaz and, and Kayak, 2009); Kis- Balaton (Lőkkös, 2014); Croatia (Turić et al., 2017); Kuwait (Edmonds et al., 2019); South Korea (Jung et al., 2020).

\section{2- Family, Gyrinidae}

Genus, Dineutus Macleay, 1825

\section{Dineutus grandis (Klug J.C.F., 1834)}

Synonym: Gyrinus grandis Klug J.C.F., 1834

Material examined: 3 specimens, Thi- Qar Province, Al-Chibayish Marshes, 19.vi.2019.

Distribution: Iraq (Ali, 1978b). North-east Africa and the Arabian Peninsula (Brinck, 1955).

Genus, Gyrinus Geoffroy, 1762

Synonyms: Gyradelphus Gozis, 1915

Gyrinidius Guignot, 1951

Gyrinoides Guignot, 1948

Gyrinulus Zaitzev, 1907

Gyrinus Linnaeus, 1767

Gyrinus Müller, 1764

Neogyrinus Hatch, 1925

Oreogyrinus Ochs, 1935

Gyrinus natator (Linnaeus, 1758)

Synonyms: Dytiscus natator Linnaeus, 1758

Gyrinus mergus Ahrens, 1812 
Al-Saffar and Augul

Material examined: 3 specimens, Thi- Qar Province, Al-Chibayish Marshes, 19.vi.2021.

Distribution: Iraq (Ali, 1978); Ireland (Nelson et al., 2019).

\title{
3- Family, Hydraenidae
}

Genus, Hydraenida Germain, 1901

Hydraenida sp.

Material examined: 2 specimens, Maysan Province: Hawizeh Marshes, Umm An-Ni'aaj, 23.viii.2021.

Distribution: Iraq (Abdulhasan et al., 2009); Chile (GBIF Secretariat, 2021).

Genus, Ochthebius Leach, 1815

Synonyms: Asiobates Thomson, 1859

Asiobathes Kuhnt, 1912

Homalochthebius Kuwert, 1887

Lunzochthebius Ienistea, 1988

Mimasiobates Ienistea, 1988

Ochtebius Thomson, 1859

Trymochthebius Kuwert, 1887

\section{Ochthebius sp.}

Material examined: 11 specimens, Maysan Province: Hawizeh Marshes, Umm An-Ni'aaj, 23.viii.2021.

Distribution: The members of the genus Ochthebius distribute in the Palaearctic, Oriental, Neotropical, Nearctic, Australian and Afrotropical regions (Villastrigo et al., 2019).

\author{
4- Family, Hydrophilidae \\ Genus, Enochrus Thomson, 1859 \\ Synonyms: Agraphilhydrus Everts, 1898 \\ Agraphilydrus Kuwert, 1888 \\ Agraphophilydrus Zaitzev, 1908 \\ Farana Knisch, 1922 \\ Holcophilhydrus d'Orchymont, 1919 \\ Holcophilhydus Orchymont, 1919 \\ Holcophilydrus Knisch, 1911 \\ Hydatotrephis MacLeay, 1871 \\ Hydatotrophis d'Orchymont, 1919 \\ Hydrotrephis Régimbart, 1908 \\ Lumetus Zaitzev, 1908 \\ Methydrus Rey, 1885 \\ Philhydrus Brullé, 1835 \\ Philydrus Solier, 1834 \\ Pseudenochrus Lomnicki, 1911
}




\section{Survey of insects}

Enochrus melanocephalus (Olivier, 1793)

Synonyms: Enochrus italus Kuwert, 1890

Hydrobius atricapillus Stephens, 1829

Hydrophilus bicolor Gyllenhal, 1808

Hydrophilus melanocephalus A. G. Olivier, 1793

Hydrophilus striatus Turton, 1802

Material examind: 3 specimens, Maysan Province: Hawizeh Marshes, Umm An-Ni'aaj, 29.viii.2020.

Distribution: Iraq (Abdulhasan et al., 2009); Kis- Balaton (Lökkös, 2014); Croatia (Turić et al., 2017); Ireland (Nelson et al., 2019).

Genus, Paracymus C. G. Thomson, 1867

Synonyms: Eumetacymus Brethes, 1922

Paracymorphus Kuwert, 1888

Quasiparacymus Marjanian, 2009

\section{Paracymus sp}

Material examined: 1specimen, Maysan Province: Hawizeh Marshes, Umm An-Ni'aaj, 23.viii.2021.

Distribution: Iraq (Abdulhasan et al., 2009). The genus of Paracymus distribute in Australia, Guyana, Argentina, Colombia, Paraguay, Venezuela, Canada, USA, France, Germany, Netherlands, Sweden, Spain, Suriname and Vietnam (GBIF Secretariat, 2021).

\section{5- Family, Georissidae}

Genus, Georissus Latreille, 1809

Synonyms: Cathammistes Illiger, 1807

Georyssus Latreille, 1809

Georyssus Stephens, 1828

Neogeorissus Satô, 1972

Nipponogeorissus Satô, 1972

\section{Georissus sp.}

Material examined: 1 specimen, Thi- Qar Province, Al-Chibayish marsh, 19.vi.2021.

Distribution: Iraq (Abdulhasan et al., 2009); this genus is worldwide distribution (GBIF

Secretariat, 2021).

\section{6- Family, Hygrobiidae}

Genus, Hygrobia Latreille, 1804

Synonyms: Hydrachna Fabricius, 1801

Hydrachne Latreille, 1802

Hygriobia Latreille, 1804

Paelobius Schonherr, 1808

Pelobius Erichson, 1832

Paelobius Schonherr, 1808 


\section{Al-Saffar and Augul}

Pelobius Erichson, 1832

Pelobius Schoenherr, 1808

Poelobius Schönherr, 1808

\section{Hygrobia sp.}

Material examined: 1 specimen, Thi- Qar Province, Al-Chibayish marsh, 19.vi.2021.

Distribution: Iraq (Abdulhasan et al., 2009); the genus of Hygrobia is native in Europe, North Africa, China and Australia (Nilsson, 2006).

\section{7- Family, Noteridae}

Genus, Canthydrus Sharp, 1882

Canthydrus luctuosus (Aubé, 1838)

Material examined: 3 specimens, Thi- Qar Province, Al-Chibayish Marshes, 19.vi.2021.

Distribution: Iraq (Abdul- Karim, 1978); Cambodia, India, Indonesia, Iran, Sri Lanka, Syria, Vietnam (Nilsson, 2011).

Genus, Hydrocanthus Say, 1823

Synonyms: Allocanthus Guignot, 1948

Sternocanthus Guignot, 1948

\section{Hydrocanthus sp.}

Material examined: 1 specimen, Thi- Qar Province, Al-Chibayish Marshes, 19.vi.2021.

Distribution: Wildly distributed (Young, 1985); Iraq (Abdulhasan et al., 2009).

Genus, Noterus Clairville, 1806

Noterus clavicornis (De Geer, 1774)

Synonyms: Dytiscus clavicornis De Geer, 1774

Dytiscus semipunctatus Fabricius, 1792

Dytiscus sparsus Marsham, 1802

Noterus convexiusculus Reiche \& Saulcy, 1855

Material examined: 2 specimens, Thi- Qar Province, Al-Chibayish marsh, 19.vi.2021.

Distribution: Iraq (Abdul-Karim, 1978); Turkey (Darilmaz and kayak, 2009); Croatia

(Turić et al., 2011); Kis- Balaton (Lőkkös, 2014); Iran (Taher and Heydarnejad, 2019).

Noterus ponticus Sharp, 1882

Material examined: 3 specimens, Thi- Qar Province, Al-Chibayish marsh, 19.vi.2021. Distribution: Iraq (Abdul-Karim, 1978); Iran (Nilsson, 2011); Kuwait (Edmonds et al., 2019).

Genus, Suphisellus Crotch, 1873

Suphisellus sp. 
Material examined: 7 specimens, Thi- Qar Province, Al-Chibayish marsh, 19.vi.2021. Distribution: Worldwide (Nilsson, 2011).

\section{(B) Order, Diptera}

1- Family, Chaoboridae

Genus, Chaoborus Lichtenstein, 1800

Synonyms: Corehtra Angelin, 1820

Corethra Meigen, 1803

Culicites Heyden, 1862

Proboscistoma Saccardo, 1864

\section{Chaoborus sp.}

Material examined: 13 specimens (larval stages); 3 specimens, Maysan Province: Hawizeh Marshes, Umm An-Ni'aaj, 31.vi.2019; 10 specimens, Thi- Qar Province, AlChibayish Marshes, 3.vii.2021.

Distribution: The genus Chaoborus is worldwide distribution (except Antarctica) (Borkent, 1993); Iraq (Abdulhasan et al., 2009).

\section{2-Family, Chironomidae}

Genus, Chironomus Meigen, 1803

Synonyms: Camptochironomus Kieffer, 1918

Chaetolabis Townes, 1945

Cheironomus Wollaston, 1858

Chiotonomus Wiedemann, 1817

Holtedahlia Kieffer, 1922

Lobochironomus Ryser, Scholl \&Wülker, 1985

Tendipes Meigen, 1800

Chironomus annularius Kieffer, 1926

Synonyms: Chironomus absconditus Kieffer, 1926

Chironomus horni Kieffer, 1918

Material examined: (9 adults, 4 larvae): Thi- Qar Province, Al- Hammar Marshes, 3.vii.2021.

Distribution: Iraq (Al-Saffar, 2008); Nearctic Region (Oliver et al., 1990); Italy (Rossaro et al., 2019)

\section{Chironomus riparius Meigen, 1804}

Synonyms: Chironomus albistria Walker, 1848

Chironomus albistris Walker, 1848

Chironomus bifilis Thienemann \& Kieffer, 1916

Chironomus curtibarba Kieffer, 1922

Chironomus curtiforceps Thienemann \& Kieffer, 1916

Chironomus dichromocerus (Kieffer, 1911)

Chironomus gregarius Kieffer, 1909 
Al-Saffar and Augul

Chironomus halochares Kieffer, 1915

Chironomus ichtyobrota (Kieffer, 1911)

Chironomus indivisus (Kieffer, 1911)

Chironomus interruptus Kieffer, 1909

Chironomus kochianus Kieffer \& Thienemann, 1919

Chironomus militaris Johannsen, 1937

Chironomus pentatomus Kieffer, 1909

Chironomus serus Malloch, 1915

Chironomus subproductus (Kieffer, 1911)

Chironomus subriparius Kieffer, 1918

Chironomus thummi (Kieffer, 1911)

Chironomus zonulus Zetterstedt, 1838

Tendipes dichromocerus Kieffer, 1911

Tendipes ichtyobrota Kieffer, 1911

Tendipes indivisus Kieffer, 1911

Tendipes rhyparobius Kieffer, 1911

Tendipes subproductus Kieffer, 1911

Tendipes thummi Kieffer, 1911

Material examined: 15 specimens: Thi- Qar Province, Al-Chibayish Marshes, 3.vii.2021. Distribution: Iraq (Abdulhasan et al., 2009); Japan (Sasa, 1989) Nearctic Region (Oliver et al., 1990); Italy (Rossaro et al., 2019); Iran (Aydın and Samin, 2020).

Genus, Glyptotendipes Kieffer, 1913

Synonyms: Caulochironomus Heyn, 1993

Heynotendipes Spies \& Saether, 2004

Phytotendipes Goetghebuer, 1937

Trichotendipes Heyn, 1993

\section{Glyptotendipes $\mathrm{sp}$}

Material examined: 13 specimens (5 adults, 8 larvae); Thi- Qar Province, Al-Chibayish Marshes, 3.vii.2021.

Distribution: Iraq (Abdulhasan et al., 2009); The genus Glyptotendipes has a Holarctic distribution (Catalogue of Life, 2009).

Genus, Paratendipes Kieffer, 1911

Synonym: Synparatendipes Thienemann, 1913

Paratendipes albimanus (Meigen, 1818)

Synonyms: Chironomus albimanus Meigen, 1818

Chironomus annularis Meigen, 1804

Chironomus heteropus Kieffer, 1906

Paratendipes annularis (Meigen, 1804)

Material examined: 7 specimens; Thi-Qar Province, Al-Chibayish Marshes, 3.vi.2019. 
Distribution: Iraq (Abdulhasan et al., 2009); Nearctic Region (Oliver et al., 1990); Korea (Na et al., 2010); Polish (Larocque- Tobler, 2014); Turkey (Aydin and Güher, 2017); Italy (Rossaro et al., 2019); Wisconsin (Egan et al., 2019); Iran (Mohammadi et al., 2021).

Genus, Pentaneura Philippi, 1865

Pentaneura sp.

Material examined: 16 specimens (7 adults, 9 larvae); Thi- Qar Province, Al-Chibayish Marshes, 3.vi.2019.

Distribution: Iraq (Abdulhasan et al., 2009); Colombia, Canda, USA, Braszil, Argentina, Indonesia, South Africa, Zimbabwe, Mozambeque and Zambia (GBIF Secrterate, 2021).

\section{3- Family, Culicidae}

Genus, Anopheles Meigen, 1818

Synonym: Baimaia Harbach, Rattanarithikul \& Harrision, 2005

Anopheles sp.

Material Examined: 20 specimens ( 8 adults, 12 larval stages); 13 specimens ( 3 adults, 10 larvae), Maysan Province: Hawizeh Marshes, Umm An-Ni'aaj, 31.vi.2019; 7 specimens (4 adults, 3 larvae), Thi- Qar Province, Al-Chibayish Marshes, 3.vii.2021.

Distribution: Iraq (Abdulhasan et al., 2009); worldwide distribution (Harbach and Kitching, 2016).

(C) Order, Ephemeroptera

1- Family, Baetidae

Genus, Procloeon Bengtsson, 1915

Synonyms: Monilistylus Kluge, 2020

Pseudocloëon Bengtsson, 1914

\section{Procloeon sp.}

Material examined: 15 specimens (3 adults, 12 nymph stages); 1 adult, 4 nymphs, Maysan Province: Hawizeh Marshes, Umm An-Ni'aaj, 31.v.2019; 2 adults, 8 nymphs, Thi- Qar Province, Al-Chibayish Marshes, 3.v.2021.

Distribution: This genus is distributed throughout the world; with excluding four species are recorded from the Oriental Region: P. debilis (Walker, 1860) from India (Kimmins, 1960); P. tatualis Waltz\& McCafferty, 1985 from Taiwan (Waltz and McCafferty, 1985); $P$. regularum Müller-Liebenau \& Hubbards, 1985 from Sri Lanka (Müller-Liebenau and Hubbard, 1985); and P. spinosum Nguyen \& Bae, 2006 from Vietnam (Tungpairojwong et al., 2006). In Iraq this genus registered by (Abdulhasan et al., 2009).

\section{2- Family, Caenidae}

Genus, Caenis Stephens, 1835

Synonyms: Austrocaenis Barnard, 1932 


\section{Al-Saffar and Augul}

Caeneus Eaton, 1888

Caenodes Ulmer, 1924

Caenomedea Thew, 1960

Caenus Agassiz, 1846

Ordella Campion, 1923

Oxycypha Burmeister, 1839

Pseudocaenis Soldan, 1978

\section{Caenis sp.}

Material examined: 8 nymph specimens; Thi- Qar Province, Al-Chibayish Marshes, 3.v.2021.

Distribution: Iraq (Abdulhasan et al., 2009). Holarctic Region (Menetrey et al., 2008).

\section{(D) Order, Hemiptera}

1- Family, Belostomidae

Genus, Belostoma Latreille, 1807

Synonyms: Belostomum Burmeister, 1835

Perthostoma Leidy, 1847

Zaitha Amyot \& Serville, 1843

Belostoma sp.

Material examined: 6 specimens; Maysan Province, Hawizeh Marshes, Umm An-Ni'aaj, 9.vii.2016

Distribution: Iraq (Ali et al., 2007); New World and Neotropical Region (Schuh and Weirauch, 2020).

Genus, Lethocerus Mayr, 1853

Synonyms: Amorgius Stål, 1866

Iliastus Gistel, 1847

Lethocerus patruelis (Stål, 1854)

Synonyms: Belostoma cousinis Stl, 1854

Lethocerus persicus (Montandon, 1898)

Material examined: 4 specimens; Thi-Qar Province, Al-Chibayish Marshes, 19. ix.2019. Distribution: Iraq (Linnavuori, 1994); Known from the Balkan Peninsula and SW Asia to SE Asia (Linnavuori, 1994), Croatia (Kment and Beran, 2011), Israel (Novoselsky et al., 2018), Kuwait (Amr, 2021).

\section{Lethocerus sp.}

Material examined: 1specimens; Thi-Qar Province, Al-Chibayish Marshes, 19.ix.2019. Distribution: It is distributed in tropical and subtropical also in temperate regions of the world; high divesity occurs in the Americas, with only one species in Europe, two in Africa, three in Asia and two in Australia (Lauck and Menke, 1961; Perez-Goodwyn, 2006). 


\section{2- Family, Corixidae}

Genus, Corisella Lundblad, 1928

\section{Corisella sp.}

Material examined: 5specimens; Thi-Qar Province, Al-Chibayish Marshes, 19.ix.2019. Distribution: Nearctic Region: USA and Canada (Hanson et al., 2007); Iraq (Abdulhasan et al., 2009); Iran (Ahmadi et al., 2011).

Genus, Micronecta Kirkaldy, 1897

Synonyms: Micronectella Lundblad, 1933

Unguinecta Nieser, Chen \& Yang, 2005

Micronecta isis Horváth, 1899

Material examined: 3specimens; Maysan Province, Hawizeh Marshes, Umm An-Ni'aaj, 9.vii.2016.

Distribution: Widespread in the Ethiopian Region, also known from Egypt, the Arabian Peninsula, Iraq and Israel (Linnavuori, 1994).

\section{Micronecta sp.}

Material examined: 1 specimen, Maysan Province, Hawizeh Marshes, Umm An-Ni'aaj, 19.vii.2019.

Genus, Sigara Fabricius, 1775

Synonym: Sigera Schellenberg, 1800

Sigara lateralis (Leach, 1818)

Synonyms: Arctocorisa hieroglyphica (Dufour, 1833)

Corisa hieroglyphica Dufour, 1833

Corixa hieroglyphica (Dufour, 1833)

Corixa lateralis Leach, 1818

Sigara hieroglyphica (Dufour, 1833)

Material examined: 11 specimens, Thi- Qar Province, Al-Chibayish Marshes, 29.viii.2020.

Distribution: Iraq (Jaczewski, 1964); Kuwait (Al-Houty, 2011); Croatia (Kment and Beran, 2011); Iran (Ghahar, 2013); Ukraine (Grandova, 2013); Hungary (Boda et al., 2015); Slovakia (Klementova et al., 2015).

Sigara septemlineata (Paiva, 1918)

Synonym: Corixa septemlineata Paiva, 1981

Material examined: 3 specimens, Thi- Qar Province, Al-Chibayish Marshes, 29.viii.2020. Distribution: Iraq (Geraci et al., 2011); Burma, Thailand, Vietnam, China, Taiwan, Korea, Russia and Japan (Insectomania, 2020).

\section{3-Family, Gerridae}




\section{Al-Saffar and Augul}

\section{Genus, Gerris Fabricius, 1794}

Synonyms: Gerriselloides Hungerford \& Matsuda, 1958

Limnotrechus Stål, 1868

\section{Gerris sp.}

Material examined: 5 specimens, Thi-Qar Province, Al-Chibayish Marshes, 29.viii.2020.

Distribution: Iraq (Ali et al., 2007); Worldwide (Henry and Froeschner, 1988).

\section{4-Family, Macrovellidae}

Genus, Macrovelia Uhler, 1872

Macrovelia hornii Uhler, 1872

Material examined: 7 specimens; Thi- Qar Province, Al-Chibayish Marshes, 29.viii.2020.

Distribution: Iraq (Al-Edani and Kareem, 2015); USA (GBIF Secretariat, 2021).

\section{5-Family: Mesovillidae}

Genus, Mesovelia Mulsant \& Ray, 1852

Synonym: Fieberia Jakovlev, 1873

Mesovelia furicata Mulsant \&Ray, 1852

Synonyms: Fieberia lacustris Jakovlev, 1873

Mesovelia fuscata Mulsant \& Rey, 1852

Mesovelia parra J. Sahlberg, 1875

Microvelia fuscata Mulsant \& Rey, 1852

Material examined: 1 specimen; Thi-Qar Province, Al-Chibayish Marshes, 29.viii.2020.

Distribution: Iraq (Abdulhasan et al., 2009); Australia (Andersen and Weir, 2004); Ukraine (Grandova, 2013); Hungary (Boda et al., 2015); Slovakia (Klementova et al., 2015); Croatia(Turić et al., 2017).

Mesovelia vittigera Horváth, 1895

Synonyms: Mesovelia orientalis Kirkaldy, 1901

Mesovelia proxima Schouteden, 1905

Material examined: 9 specimens; Maysan Province, Hawizeh Marshes, Umm An-Ni'aaj, 9.vii.2016.

Distribution: Iraq (Al-Edani and Kareem, 2015); Croatia (Kment and Beran, 2011); Philippines (Zettel, 2014).

\section{6-Family: Notonectidae}

\section{Genus, Buenoa Kirkaldy, 1904}

\section{Buenoa sp.}

Material examined: 2 specimens; Thi- Qar Province, Al-Chibayish Marshes, 29.viii.2020. Distribution: Iraq (Abdulhasan et al., 2009); Colombia, USA, Argentine, Mexico, Jamaica, Martinique, Costa Rica (GBIF Secretariat, 2021). 
7-Family, Pleidae

Genus, Plea Leach, 1817

Plea minutissma (Leach, 1817)

Synonym: Plea leachi McGregor \& Kirkaldy, 1899

Material examined: 12 specimens; Thi- Qar Province, Al-Chibayish Marshes, 29.viii.2020.

Distribution: Liberian Peninsula (Garrido and Munilla, 2008); Iraq (Abdulhasan et al., 2009); Croatia (Kment and Beran, 2011); Ukraine (Grandova, 2013); Hungary (Boda et al., 2015); Slovakia (Klementova et al., 2015 ).

\section{(E) Order, Hymenoptera}

1- Family, Apidae

Genus, Apis Linnaeus, 1758

Synonyms: Apiarus Rafinesque, 1815

Apicula Rafinesque, 1814

Apis mellifera Linnaeus, 1758

Synonyms: Apis adansonii Latreille, 1804

Apis aenigmaticus Rayment, 1925

Apis australis Kiesenwetter, 1860

Apis caffra Lepeletier, 1836

Apis cerifera Gerstäcker, 1862

Apis cerifera Scopoli, 1770

Apis daurica Fischer von Waldheim, 1843

Apis fasciata Latreille, 1804

Apis gregaria Geoffroy, 1762

Apis intermissa Maa, 1953

Apis mellifica Linnaeus, 1761

Apis nigritarum Lepeletier, 1836

Apis siciliana Dalla Torre, 1896

Apis sicula Montgano, 1911

Materials Examined: 18 specimens, Maysan Province, Hawizeh Marshes, Umm AnNi'aaj, 23.vii.2020.

Distribution: This species is most widespread, occurring throughout Europe, Africa, Northern Western Asia, Caucasia, and the Iranian Plateau (Ruttner, 2003; Sheppard and Meixner, 2003), as well as adventives in the Americas and Australia (Kerr, 1957; Moritz et al., 2005).

Genus, Xylocopa Latreille, 1802

Synonyms: Hylocopa Kirchner, 1857

Xilocopa Latreille, 1802

Xylocopa fenestrata (Fabricius, 1798) 


\section{Al-Saffar and Augul}

Material examined: 7 specimens, Maysan Province, Hawizeh Marshes, Umm An-Ni'aaj, 19.vi.2020.

Distribution: Turkey (Wancke, 1982); Iraq (Derwesh, 1965); Syria, Iran, Pakistan, Nepal India, Israel, Burma, China, Madagascar, Reunion (Guershon and Ionescu-Hirsch, 2012).

\section{2-Family, Megachilidae}

Genus, Coelioxys Latreille, 1809

Synonyms: Caelioxys Say, 1824

Coelioxis Germar, 1817

Coelioxys haemorrhoa Förster, 1853

Materials examined: 5 specimens, Maysan Province: Hawizeh Marshes, Umm An-Ni'aaj, 31.v.2020.

Distribution: Iraq (Derwesh, 1965). Southern Europe, from the Iberian Peninsula to Austria, and North Africa, to Central Asia (Ornosa et al., 2007).

\section{3- Family, Scoliidae}

Genus, Campsomeriella Betrem, 1941

Campsomeriella thoracica (Fabricius, 1787)

Synonym: Scolia thoracica Fabricius, 1787

Material Examined: 3 specimens, Maysan Province: Hawizeh Marshes, Umm An-Ni'aaj, 29.vi.2020.

Distribution: Saudi Arabia (Shalaby, 1961); Iran (Chahartaghi Abineh, 2002); Oman (Osten, 2005a); Crete, Cyprus, Dodecanese Is., Greek mainland, Italian mainland, Malta, North Aegean Is., North Africa, Sicily, Spanish mainland, Syria, Turkey (Fallahzadeh and Saghaei, 2010); Iraq (Khalaf, 1959).

Genus, Scolia J.C. Fabricius, 1775

Synonyms: Ascoli Guérin-Méneville, 1838

Lacosi Guérin-Méneville, 1838

Solia Dalla Torre, 1897

\section{Scolia turkestanica Betrem, 1935}

Material examined: 1 specimen, Maysan Province: Hawizeh Marshes, Umm An-Ni'aaj, 29.vi.2020.

Distribution: Armenia, Iran, Iraq, Turkey, Turkmenistan and Uzbekistan (Steinberg, 1962); Kirgizstan and Tadzhikistan (Osten, 2005 b).

Scolia flaviceps Eversmann, 1846

Materials examined: 11 specimens, Maysan Province, Hawizeh Marshes, Umm AnNi'aaj, 18.v.2020.

Distribution: Afghanistan, Iran, Iraq, Oman (Betrem, 1935); Turkey (Madl, 1997); Crete, Tadzhikistan, Turkmenistan, Uzbek.istan, Central Asia, Cyprus (Osten, 1999); France, 


\section{Survey of insects}

Italy, Egypt (Osten, 2000); Saudi Arabia and United Emirates (Osten et al., 2003); Greece (Osten and Arens, 2004).

\section{4- Family, Vespidae}

Genus, Delta Saussure, 1855

Synonyms: Erinys Zirngiebl, 1953

Phi Saussure, 1855

Delta esuriens (Fabricius, 1787)

Synonym: Eumenes esuriens Fabricius, 1804

Material examined: 11 specimens; Maysan Province: Hawizeh Marshes, Umm An-Ni'aaj, 1. xi.2021.

Distribution: Iraq (Vecht and Fischer, 1972); India, Indonesia, Iran, Myanmar, Pakistan, Philippines, Saudi Arabia, Sri Lanka; Thailand, Vietnam (Nguyen et al., 2007); Thailand (Srinivasan and Kumar, 2010); Australia, Laos, Mauritius, Timor (Kumar, 2012).

Genus, Polistes Latreille, 1802

Synonyms: Eupolistes Dalla Torre, 1904

Leptpolistes Bluthgen, 1943

Polistula Weyrauch, 1938

Polistus Latreille, 1804

Pseudopolistes Weyrauch, 1937

Sulcopolistes Bluthgen, 1938

Polistes wattii Cameron, 1900

Material Examined: 2 specimens, Maysan Province: Hawizeh Marshes, Umm An-Ni'aaj, 1.xi.2020.

Distribution: Afghanistan, China, India, Iraq, Iran, Oman, Pakistan, Mauritius, Saudi Arabia and United Arab Emirates (Das and Gupta, 1989; Carpenter, 1996); Kazakhstan (Kazenas, 2014); Tajikistan (Castro and Dvorak, 2010), and Turkmenistan (Dubatolov, 2019).

Genus, Vespa Linnaeus, 1758

Synonym: Macrovespa Dalla Torre, 1904

Vespa orientalis Linnaeus, 1761

Synonyms: Vespa aegyptiaca André, 1884

Vespa aegyptiaca Vallot, 1802

Vespa fusca Christ, 1791

Vespa indica Wrought., 1889

Vespa jurinei de Saussure, 1854

Vespa quadripunctata Forskal, 1775

Vespa turcica Drury, 1773

Material examined: Maysan Province: Hawizeh Marshes, Umm An-Ni'aaj, 1.xi.2016. 
Al-Saffar and Augul

Distribution: Iraq (Morice, 1921); Northern part of Africa, Southeastern Europe, Southwest Asia across Turkey and Arabian Peninsula to India, and Nepal (Carpenter and Kojima, 1997; Archer, 1998); Mexico (Dvorák, 2006).

Genus, Odynerus Latreille, 1802

Synonyms: Epipone Kirby \& Spence, 1815

Euepipona Dalla Torre, 1904

Hoplomerus Agassiz, 1846

Hoplopus Agassiz, 1846

Oplomerus Westwood, 1840

Oplopus Wesmael, 1836

Odynerus spinipes (Linnaeus, 1758)

Synonyms: Hoplomerus scutellaris Blüthgen, 1940

Odynerus amurensis Blüthgen, 1941

Odynerus flavicapus Mader, 1936

Odynerus flaviscapus Mader, 1936

Odynerus muticus Zetterstedt, 1839

Vespa quinquefasciata Fabricius, 1793

Material examined: 3 specimens; Maysan Province: Hawizeh Marshes, Umm An-Ni'aaj, 30.x.2020.

Distribution: Europe, Turkey (Yıldırım and Kojima, 1999); this species is recorded in Iraq under the name Hoplomerus spinipes (Linnaeus, 1758) by Khalaf (1958).

Genus, Stenodynerus Saussure, 1863

\section{Stenodynerus sp.}

Material examined: 2 specimens, Maysan Province: Hawizeh Marshes, Umm An-Ni'aaj, 19.vi.2020.

Distribution: The genus of Stenodynerus distributes spreads along the Nearctic, Palearctic, Oriental and Neotropical regions (Carpenter, 1986).

\section{(F) Order: Lepidoptera}

1- Family: Crambidae

Genus, Parapoynx Hübner, 1825

Synonyms: Eustales Clemens, 1860

Microdracon Warren, 1890

Nymphaeella Grote, 1880

Parapoynx fluctuosalis (Zeller, 1852)

Synonyms: Nymphula fluctuosalis Zeller, 1852

Oligostigma chrysippusalis Walker, 1859

Oligostigma curta Butler, 1879

Oligostigma obitalis Walker, 1859 
Parapoynx chrysippusalis (Walker, 1859)

Parapoynx curta (Butler, 1879)

Parapoynx linealis Guenée, 1854

Parapoynx obitalis (Walker, 1859)

Parapoynx oryzalis Wood-Mason, 1885

Material examined: 2 specimens, Thi- Qar Province, Al-Chibayish Marshes, 1.vi.2021. Distribution: Oriental region (Speidel and Mey, 1999); China (Chen et al., 2006); Iraq (Abdulhasan et al., 2009); Africa (Agassi, 2012); Mauritius (Bippus, 2019 ); widespread in continental Africa, Southern Asia, Southern Palearctic region to Japan, Australasia and Pacific islands, also recorded from Puerto Rico" (de Prins and de Prins, 2019); India (Alex et al., 2021).

\section{2- Family, Lycaenidae}

Genus, Polyommatus Latreille, 1804

Polyommatus baeticus (Linnaeus, 1767)

Synonyms: Cosmolyce baeticus (Linnaeus, 1767)

Lycaena baetica (Linnaeus, 1767)

Papilio baeticus Linnaeus, 1767

Material Examined: 2 specimens, Maysan Province: Hawizeh Marshes, Umm AnNi'aaj, 3.vi.2020.

Distribution: Southern Europe, Africa, Tropical and Subtropical Asia, Australia (Forster, 1963); Iraq (Derwesh, 1965).

\section{(G) Order, Odonata}

1- Family, Aeshnidae

Genus, Aeshna Fabricius, 1775

Synonyms: Aeschna Fabricius, 1775

Aeschna Illiger, 1801

Oeshna Lamarck, 1817

Aeshna mixta Latreille, 1805

Synonyms: Aeschna alpina Selys, 1848

Aeschna habermayeri Götz, 1923

Aeschna mixta Latreille, 1805

Aeshna alpina Selys, 1848

Aeshna coluberculus Harris, 1782

Aeshna habermayeri Götz, 1923

Aeshna lucia Needham, 1930

Libellula coluberculus Harris, 1782

Material examined: 12 specimens (4 adults, 8 Naiads); Thi- Qar Province, Al-Chibayish Marshes, 3.vii.2021.

Distribution: Iraq (Morton, 1919), Garisticki and Amr (2011) listed this species in Iraqi Marshes; Europe, Asia Minor and Central Asia, reaching Kashmir in the east, and North 


\section{Al-Saffar and Augul}

Africa (Dumont, 1991; Steinmann, 1997); Iran (Heidari and Dumont, 2002); Turkey

(Salur and Özsaraç, 2004); Mediterranean and North Africa (Boudot et al., 2009).

Genus, Anax Leach, 1815

Anax ephippiger (Burmeister, 1839)

Synonyms: Aeschna ephippigera Burmeister, 1839

Aeshna mediterranea Selys, 1839

Anax senegalensis Rambur, 1842

Anax marginope Baijal \& Agarwal, 1955

Material examined: 3 specimens (adults), Thi- Qar Province, Al-Chibayish Marshes, 3.vii.2021.

Distribution: Iraq (Morton, 1920) and listed this species in Iraqi Marshes. Garisticki and Amr (2011); Turkestan and India to South Africa and Madagascar south-central Europe (Steinmann, 1997); Cyprus (De Knijf and Demolder 2013); Turkey (Hacet, 2017).

Anax imperator Leach, 1815

Synonyms: Aeschna azurea Charpentier, 1825

Aeschna formosa Vander Linden, 1823

Aeschna lunata Kolenati, 1856

Aeshna formosa Vander Linden, 1823

Anax azurea Charpentier, 1825

Anax dorsalis Burmeister, 1839

Anax formosus Vander Linden, 1820

Anax lunatus Kolenati, 1856

Material examined: 7 specimens (adults); Thi-Qar Province, Al-Chibayish Marshes, 3.vii.2021.

Distribution: Iraq (Asahina 1973, and listed in Iraqi Marshes (Abdulhasan et al., 2009); Turkey (Kalkman, 2006); Africa and through most of Europe, Arabian Peninsula, and south-western and central Asia (Mitra, 2016); Palestine (Adawi et al., 2017); Pakistan (Akbar et al., 2017); Iran (Schneideret et al., 2018).

Anax parthenope Selys, 1839

Synonyms: Aeschna parthenope Selys, 1839

Aeshna parthenope Selys, 1839

Anax bacchus Hagen, 1867

Anax major Götz, 1923

Anax parisinus Rambur, 1842

Material examined: 5 specimens: Thi- Qar Province, Al-Chibayish Marshes, 3.vii.2021.

Distribution: Iraq (Morton, 1920), Garisticki and Amr (2011) reported listed this species for Iraqi Marshes. Jordon (Katebah- Bader et al., 2004); Hong Kong (Reels 2010); It is ranges from Europe and North Africa to the Arabian Peninsula, Siberia, India, China and Japan. In the West of Europe, also found now in Latvia, Poland, south of Sweden, 
northern Germany and East of Ireland (Mitra and Clausnitzer, 2018); Kuwait (Amr, 2021).

\section{2- Family, Calopterygidae}

Genus, Calopteryx Leach, 1815

Calopteryx splendens Harris, 1780

Synonyms: Agrion parthenias Charpentier, 1840

Agrion splendens (Harris, 1780)

Calopteryx ludoviciana Leach, 1815

Calopteryx ludoviciana Selys, 1840

Calopteryx parthenias Charpentier, 1840

Calopteryx shachrudica Bartenef, 1916

Coenagrion splendens (Harris, 1780)

Libellula splendens Harris, 1780

Material examined: 3 adult specimens, Thi- Qar Province, Al-Chibayish Marshes, 31.iv.2021.

Distribution: Widely distributed in Palearctic Region (Sadeghi et al., 2010). Turkey (Salur and Özsaraç, 2004); Georgia (Schröter, 2010); Iraq (Gastecki and Amr, 2011); Iran (Schneideret et al., 2018).

\section{3- Family, Coenagriidae}

Genus, Ischnura Charpentier, 1840

Synonyms: Anomalagrion Selys, 1857

Ischnosoma Wallengren, 1894

Nanosura Kennedy, 1920

Ischnura elegans Vander Linden, 1820

Synonyms: Agrion aglae Boyer de Fonscolombe, 1838

Agrion elegans Vander Linden, 1820

Agrion hastulatum Burmeister, 1839

Agrion pupilla Hansemann, 1823

Ischnura aglae Fonscolombe, 1838

Ischnura aurantiaca Roster, 1886

Ischnura excelsa Roster, 1886

Ischnura exigua Roster, 1886

Ischnura ezonata Stephens, 1836

Ischnura hastulata Burmeister, 1839

Ischnura infuscans Campion \& Campion, 1905

Ischnura lamellata Kolbe, 1885

Ischnura magna Roster, 1886

Ischnura pupilla Hansemann, 1823

Ischnura rufescens Stephens, 1836

Ischnura tuberculata Charpentier, 1825

Ischnura zonata Stephens, 1835

Material examined: 2 adult specimens, Thi-Qar Province, Al-Chibayish Marshes, 3.vii.2021. 


\section{Al-Saffar and Augul}

Distribution: Iraq (Abdulhassan et al., 2009); It distributes from Spain in the West, to Japan in the East, and from Sweden in the North to Iran in the South (Boudot and Salamun, 2015); Georgia (Schröter, 2010); Turkey (Salur and Özsaraç, 2004).

\section{Ischnura evansi Morton, 1919}

Material examined: 3 specimens (1 naiad, 2 adults), Thi- Qar Province, Al-Chibayish Marshes, 31.iv.2021.

Distribution: Iraq Morton (1919), Garsetic and Amr (2011) listed this species in Iraqi marshes; Africa: Djibouti, Egypt and Sudan (Clausnitzer et al., 2012); Asia: Turkey (Asian part), Palestine, Jordan, Syria, Iran, UAE, Oman, Saudi Arabia, Qatar (GBIF Secretariat, 2021); Kuwait (Amr, 2021).

\section{Ischnura fountaineae Morton, 1905}

Synonyms: Ischnura bukharensis Bartenef, 1913

Ischnura fountainei Morton, 1905

Material examined: 1 adult specimen, Thi- Qar Province, Al-Chibayish Marshes, 31.iv.2021

Distribution: Iraq (Ashina, 1973), this species was listed by Garsetic and Amr (2011) in Marshes. It occurs across northern Africa from eastern Morocco to Egypt, east to Kazakhstan and westernmost China, Saudi Arabia, Qatar, United Arab Emirates and Oman (Boudot et al., 2015).

Ischnura senegalensis Rambur, 1842

Synonyms: Agrion senegalense Rambur, 1842

Agrion senegalensis Rambur, 1842

Enallagma brevispina Selys, 1876

Ischnura brevispina de Selys, 1876

Material examined: 7 adult specimens; Maysan Province: Hawizeh Marshes, Umm AnNi'aaj, 23.vi.2021.

Distribution: Iraq (Kalkman, 2009) this species was reported in Marshes by Garsetic and Amr (2011); Native from Africa, through the Middle East, to southern and eastern Asia (Sharma and Clausnitzer, 2016); Malasyia (Yen and Dawood, 2021).

\section{4-Family, Corduliidae}

Genus, Somatochlora Selys, 1871

Synonyms: Chlorosoma Charpentier, 1839

Somatachlora Brauner, 1902

\section{Somatochlora sp.}

Material examined: 1 adult specimen, Thi-Qar Province, Al-Chibayish Marshes, 31.iv.2021.

Distribution: Iraq (Abdulhasan et al., 2009); Member of this genus are found in Europe, Asia and North America (Cannings and Cannings, 1985); Turkey (Kalkman, and Wasscher, 2003); Taiwan (Zhang et al., 2014). 


\section{5- Family, Gomphidae}

Genus, Anormogomphus Selys, 1854

Anormogomphus kiritshenkoi Bartenev, 1913

Material examined: 3 adult species; Thi-Qar Province, Al-Chibayish Marshes, 29.viii.2020.

Distribution: Iraq (Morton, 1919), Garsetic and Amr (2011) registered this species in the southern marshes. Iran (Heidari and Dumont, 2002); Turkey, Syria and Iran (Kalkman, 2006 b).

Genus, Lindenia Vander Linden, 1825

Lindenia tetraphylla Vander Linden, 1825

Synonyms: Aeshna tetraphylla Vander Linden, 1825

Lindenia inkiti Bartenef, 1929

Lindenia phyllura Eichwald, 1829

Lindenia praedator; Rambur, 1842

Material examined: 6 adult specimens; Thi-Qar Province, Al-Chibayish Marshes, 29.viii.2020.

Distribution: Iraq (Garstecki and Amr, 2011); it's a wide distribution range in most parts of Central and Southwest Asia, and eastern Mediterranean (Boudot and Kalkman, 2015). It was also found Tunisia (Kunz and Kunz, 2001), Iran (Heidari and Dumont, 2002), Italy (Terzani, 2002), Algeria (Boudot et al., 2009), Bulgaria (Gastarov and Beshkov, 2010), Georgia (Schröter, 2010), Kuwait (Amr, 2021).

Genus, Onychogomphus Selys, 1854

Synonyms: Onigogomphus Brauner, 1902

Paragomphus Cowley, 1934

Onychogomphus flexuosus Schneider, 1845

Synonym: Gomphus flexuosus Schneider, 1845

Material examined: 2 adult specimens; Thi-Qar Province, Al-Chibayish Marshes, 27.viii.2020.

Distribution: Iraq (Morton, 1919) and listed from marshes (Garstecki and Amr, 2011); Palestine (Morton, 1924); Caucasus (Zazanashvili and Mallon, 2009); Georgia (Schroter et al., 2015); Turkey (Salur and Özsaraç, 2004); Armenia (Ananain and Tailly, 2013); This species distributed from Anatolia and Jordan Valley to Tajikistan and Afghanistan (Kakman et al., 2009).

6-Family, Libellulidae

Genus, Brachythemis Brauer, 1868

Brachythemis fuscopalliata Selys, 1887 


\section{Al-Saffar and Augul}

Material examined: 4 adult specimens; Maysan Province, Hawizeh Marshes, Umm AnNi'aaj, 9.vii.2016.

Distribution: Iraq (Morton, 1919), Ali et al. (2002) listed this species in Iraqi Marshes. Iran, Israel, Syria, and Turkey (GBIF Secretariat, 2021).

Genus, Crocothemis Brulle, 1832

Crocothemis erythraea Brullé, 1832

Synonyms: Crocothemis coccinea Charpentier, 1840

Crocothemis lorti Kirby, 1896

Crocothemis victoria Fourcroy, 1785

Libellula erythraea Brullé, 1832

Libellula ferruginea Vander Linden, 1825

Material examined: 2 adult species; Thi- Qar Province, Al-Chibayish Marshes, 29.viii.2020.

Distribution: Iraq (Morton, 1919), Garstecki and Amr (2011) reported this species in Iraqi Marshes. Eastern Palearctic reaching the Netherlands (Hermans and Gubbels, 1997), Belgium (Knijf, 2003), south of England (Jones, 1996), Austria (Schweiger, 1983), Croatia (Trilar and Bedjanič, 1999), France (Rehfeldt, 1991), Germany (Müller, 1987). Also this species was registered in Asian part of Western Palearctic: Jordan (Schneider, 1985), Iran (Heidari and Dumont, 2002); Kazakhstan (Chaplina, 2004), Tajikistan (Borisov, 1987), Turkey (Hacet and Aktaç, 1997), Saudi Arabia (Schneider, 1995) and northern Africa: Tunisia (Jodicke, 2003); Kuwait (Amr, 2021).

Crocothemis servilia Drury, 1773

Synonyms: Crocothemis ferruginata Fabricius, 1781

Crocothemis flavostigma Navás, 1932

Crocothemis indica Sahni, 1965

Crocothemis novaguineensis Foerster, 1898

Crocothemis reticulata Kirby, 1886

Crocothemis soror Rambur, 1842

Libellula ferruginea Fabricius, 1793

Libellula servilia Drury, 1773

Libellula soror Rambur, 1842

Material examined: 9 adult specimens; Maysan Province, Hawizeh Marshes, Umm AnNi'aaj, 9.vii.2016.

Distribution: Iraq (Morton, 1919), Garstecki and Amr (2011) reported this species in Iraqi Marshes. Iran (Heidari and Dumont, 2002); Armenia (Ananain and Tailly, 2013); its native to east and southeast Asia and introduced to Jamaica, Florida, and Hawaii (Subramanian et al., 2018); Kuwait (Amr, 2021).

Genus, Diplacodes Kirby, 1889

Diplacodes lefebvrii Rambur, 1842 


\section{Survey of insects}

Synonyms: Diplacodes concinna Rambur, 1842

Diplacodes flavistyla Rambur, 1842

Diplacodes limbata Fraser, 1949

Diplacodes morio Schneider, 1845

Diplacodes parvula Rambur, 1842

Diplacodes spinulosa Navás, 1915

Diplacodes tetra Rambur, 1842

Diplacodes unimacula Foerster, 1906

Libellula lefebvrii Rambur, 1842

Material examined: 3 adult specimens, Thi-Qar Province, Al-Chibayish Marshes, 3.vii.2021.

Distribution: Iraq (Morton, 1919), Garstecki and Amr (2011) reported this species in Iraqi Marshes. ; Iran (Heidari and Dumont, 2002); widespread and common Afrotropical species that extends to southern (KalKman et al., 2009); Africa, India, Israel, Spain, Portugal, Oman, UAE, Italy (GBIF Secretariat, 2021).

Genus, Orthetrum Newman, 1833

Orthetrum sabina Drury, 1773

Synonyms: Lepthemis divisa Selys, 1878

Libellua leptura Burmeister, 1839

Libellula ampullacea Schneider, 1845

Libellula gibba Fabricius, 1798

Libellula sabina Drury, 1770

Orthetrum ampullacea Schneider, 1845

Orthetrum divisum Selys, 1878

Orthetrum gibba Fabricius, 1798

Orthetrum leptura Burmeister, 1839

Orthetrum nigrescens Bartenef, 1929

Orthetrum viduatum Lieftinck, 1942

Material examined: 5 specimens: (4 adults); Maysan Province: Hawizeh Marshes, Umm An-Ni'aaj, 31.vi.2019; 1adult, Thi- Qar Province, Al-Chibayish Marshes, 3.vii.2021.

Distribution: Iraq (Morton, 1919), Garstecki and Amr (2011) reported this species in Iraqi Marshes. Iran (Heidari and Dumont, 2002); Armenia (Ananain and Tailly, 2013); Kuwait (Amr, 2021); Malasyia (Yen and Dawood, 2021). Australia, Indonesia, Singapore, Thailand, India, UAE, Malaysia, Papua New Guinea, China, Hong Kong, Chinese Taipei, Bangladesh, Cyprus, Macao (GBIF Secretariat, 2021).

Orthetrum taeniolatum Schneider, 1845

Synonyms: Libellula taeniolata Schneider, 1845

Orthetrum brevistylum Kirby, 1896

Orthetrum garhwalicum Singh \& Baijal, 1954

Orthetrum hyalianum Kirby, 1886

Orthetrum hyalinum Kirby, 1886 
Material examined: 2 adult specimens, Thi-Qar Province, Al-Chibayish Marshes, 3.vii.2021.

Distribution: Iraq (Morton, 1919), Garstecki and Amr (2011) reported this species in Iraqi Marshes. Its distribution spreads from Eastern Europe to China (Mitra, 2013); Kuwait (Amr, 2021).

\section{Orthetrum trinacria Selys, 1841}

Synonyms: Libellula trinacria Selys, 1841

Orthetrum bremii Rambur, 1842

Orthetrum clathrata Rambur, 1842

Material examined: 3adult specimens, Thi- Qar Province, Al-Chibayish Marshes, 3.vii.2021.

Distribution: Iraq (Morton, 1919), Garstecki and Amr (2011) reported this species in Iraqi Marshes. Iran (Heidari and Dumont, 2002); widespread in Africa and common in the north extending east to Sudan, Egypt and the Middle East. In Europe, this species distribute from Sicily and recently recorded in Sardinia and Spain (Belle, 1984; Askew, 2004).

Orthetrum chrysostigma Burmeister, 1839

Synonyms: Libellula barbara Selys, 1849

Libellula chrysostigma Burmeister, 1839

Orthetrum barbarum Selys, 1849

Material examined: 4 adult specimens; Maysan Province: Hawizeh Marshes, Umm AnNi'aaj, 31.vi.2019.

Distribution: Iraq (Augul et al., 2016 ); Algeria, Angola, Benin, Botswana, Burkina Faso, Cameroon, Chad, Congo, Côte d'Ivoire, Egypt, Eritrea, Ethiopia, Gambia, Ghana, Greece Guinea, Iran, Israel, Jordan, Kenya, Lebanon, Liberia, Libya, Malawi, Mali, Mauritania, Morocco, Mozambique, Namibia, Niger, Nigeria, Oman, Palestine, Portugal, Rwanda, Saudi Arabia, Senegal, Sierra Leone, Somalia, South Africa, South Sudan, Spain, Sudan, Syria, Tanzania, Togo, Tunisia, Turkey, Uganda, UAE, Yemen, Zambia and Zimbabwe (Boudot, 2016).

Genus, Pantala Hagen, 1861

Pantala flavescens Fabricius, 1798

Synonyms: Libellula analis Burmeister, 1839

Libellula flavescens Fabricius, 1798

Libellula terminalis Burmeister, 1839

Libellula viridula Palisot de Beauvois, 1807

Orthetrum mathewi Singh \& Baijal, 1955

Pantala analis Burmeister, 1839

Pantala mathewi Singh \& Baijal, 1954

Pantala tandicola Singh, 1955

Pantala terminalis Burmeister, 1839 
Pantala viridula Palisot de Beauvois, 1807

Sympetrum tandicola Singh, 1955

Material examined: 7 specimens (5 adults, 2 naiads); Thi- Qar Province, Al-Chibayish Marshes, 3.vii.2021.

Distribution: Iraq (Garstecki and Amr, 2011); Cyprus, Egypt, Israel, Jordon, Lebanon, Turey (Kalkman et al., 2009); Colorado (Kondarteiff and Durfee, 2010); Malasyia (Yen and Dawood, 2021); USA, Argentina, Brazil, Panama, Ecuador, Mexico, Peru, Uruguay, Bolivia, Guatemala, South Africa, Botswana, Madagascar, Congo, Zimbabwe, Zambia, Namibia, UAE, Cambodia, Thailand, China, Chinese Taipei, Hong Kong, India, Indonesia, Sir Lanka, Australia, Papua New Guinea (GBIF Secretariat, 2021).

Genus, Selysiothemis Vander Linden, 1825

Selysiothemis nigra Vander Linden, 1825

Synonyms: Libellula nigra Vander Linden, 1825

Selysiothemis advena Selys, 1878

Material examined: 1 adult specimen; Thi-Qar Province, Al-Chibayish Marshes, 3.vii.2021.

Distribution: Iraq (Morton, 1919), Garstecki and Amr (2011) listed this species in Iraqi Marshes. This species is mainly a distribution from central Asia, the Middle East and Arabia; also extends into the western Mediterranean and northern African (Boudot, 2010). Iran (Heidari and Dumont, 2002); Armenia (Ananain and Tailly, 2013); Kuwait (Amr, 2021).

Genus, Sympetrum Newman, 1833

Synonyms: Diplax Burmeister, 1839

Sympertum Brauner, 1902

Tarnetrum Needham \& Fisher, 1936

Sympetrum arenicolor Jödicke, 1994

Synonym: Sympetrum deserti Jödicke, 1994

Material examined: 4 adult specimens; Thi-Qar Province, Al-Chibayish Marshes, 3.vii.2021.

Distribution: Iraq (Kalkman, 2006; Ali and Khidhir, 2015), Garstecki and Amr (2011) listed this species in Iraqi Marshes. Afghanistan, Armenia, Azerbaijan, India, Iran, Israel, Kazakhstan, Kyrgyzstan, Syria, Tajikistan, Turkey and Turkmenistan (Malikova, 2009); Georgia (Schröter, 2010).

Sympetrum fonscolombii Selys, 1840

Synonyms: Libellula fonscolombii Selys, 1840

Sympetrum azorense Gardner, 1959

Sympetrum erythroneura Schneider, 1845

Sympetrum insignis Brittinger, 1850

Sympetrum rhaeticum Buchecker, 1873 
Al-Saffar and Augul

Tarnetrum fonscolombii (Selys, 1840)

Material examined: 4 adult specimens; Thi- Qar Province, Al-Chibayish Marshes, 3.vii.2021.

Distribution: Iraq (Morton, 1919), Garstecki and Amr (2011) registered this species in Iraqi Marshes. Afghanistan, Albania, Algeria, Angola, Armenia, Austria, Azerbaijan, Bahrain, Bangladesh, Belgium, Bhutan, Botswana, Bulgaria, Burundi, Cameroon, Chad, Croatia, Cyprus, Czech, Egypt, Eritrea, Eswatini, Ethiopia, France, Gambia, Georgia, Germany, Greece, Guinea, Hungary, India, Iran, Iraq, Israel, Italy, Jordan, Kazakhstan, Kenya, Kyrgyzstan, Latvia, Lebanon, Libya, Luxembourg, Madagascar, Mali, Malta, Mauritania, Monaco, Mongolia, Montenegro, Morocco, Mozambique, Namibia, Nepal, Netherlands, Niger, North Macedonia, Oman, Pakistan, Palestine, Poland, Portugal, Qatar, Romania, Russian Federation, Rwanda, Réunion, Saudi Arabia, Senegal, Serbia, Slovakia, Slovenia, Somalia, South Africa, Spain, Sri Lanka, Sudan, Sweden, Switzerland, Syria,Tajikistan, Tunisia, Turkey, Turkmenistan, Uganda, Ukraine, UAE, UK, Uzbekistan, Yemen, Zambia and Zimbabwe (Clausnitzer, 2013).

\section{Sympetrum striolatum Charpentier, 1840}

Synonyms: Libellula striolata Charpentier, 1840

Sympetrum neglectum Artobolevsky, 1928

Sympetrum nigrescens Lucas, 1912

Sympetrum ruficollis Charpentier, 1840

Material examined: 1 adult specimen; Thi- Qar Province, Al-Chibayish Marshes, 29.viii.2020.

Distribution: Iraq (Kalkman, 2006), Garstecki and Amr (2011) listed this species in Iraqi Marshes. Albania, Algeria, Andorra, Armenia, Austria, Azerbaijan, Belarus, Belgium, Bulgaria, China, Croatia, Cyprus, Czech, Denmark, Estonia, Finland, France, Georgia, Germany, Greece, Hungary, Iran, Ireland, Israel, Italy, Japan, Korea, Korea, Latvia, Lebanon, Lithuania, Luxembourg, Malta, Moldova, Monaco, Mongolia, Montenegro, Morocco, Netherlands, North Macedonia, Norway, Poland, Portugal, Romania, Russian Federation, Serbia, Slovakia, Slovenia, Spain, Sweden, Switzerland. Syria, Tajikistan, Tunisia, Turkey, Ukraine, United Kingdom and Uzbekistan (Clausnitzer, 2020).

Genus, Trithemis Brauer, 1868

Trithemis annulata Palisot de Beauvois, 1807

Synonyms: Libellula annulata Palisot de Beauvois, 1807

Libellula haematina Rambur, 1842

Trithemis obsoleta Rambur, 1842

Trithemis rubrinervis Selys, 1841

Trithemis violacea Sjöstedt, 1899

Material examined: 2 adult specimens; Thi-Qar Province, Al-Chibayish Marshes, 5.vii.2021.

Distribution: Iraq (Garstecki and Amr 2011); Iran (Heidari and Dumont, 2002); Kuwait (Amr, 2021). 
Trithemis festiva Rambur, 1842

Synonyms: Libellula festiva Rambur, 1842

Libellula infernalis Brauer, 1865

Trithemis cyprica Selys, 1887

Trithemis infernalis Brauer, 1865

Trithemis proserpina Selys, 1878

Trithemis prosperina Selys, 1878

Material examined: 3 adult specimens; Thi-Qar Province, Al-Chibayish Marshes, 3.vii.2021.

Distribution: Iraq (Garstecki and Amr 2011); Iran (Heidari and Dumont, 2002); Malasyia (Yen and Dawood, 2021).

\section{7- Family, Lestidae}

Genus, Sympecma Selys, 1840

Sympecma paedisca Brauer, 1877

Synonyms: Sympecma annulata Selys, 1887

Sympecma braueri Yakobson \& Bianki, 1904

Sympecma kashmirensis Ander, 1944

Sympecma striata St Quentin, 1963

Sympycna braueri Bianchi, 1904

Sympycna paedisca Brauer, 1877

Material examined: 1 adult specimens; Thi- Qar Province, Al-Chibayish Marshes, 2.vii.2021.

Distribution: Iraq (Asahina, 1973); Iran (Heidari and Dumont, 2002); Armenia (Ananain and Tailly, 2013).

\section{CONFLICTS OF INTEREST STATMENT}

"The authors have no conflicts of interest to declare".

\section{LITRETURE CITED}

Abdulhasan, N. A., Salim, M. A. A., Al-Obaidi, G. S., Ali, H. J., Al-Saffar, M. A., Abd, I. M. and Minjil, M. Sh. 2009. Habitat mapping and monitoring project classification and description of Southern Iraqi Marshlands. Nature Iraq Report, Sulaimani, Kurdistan, Iraq, $86 \mathrm{pp}$.

Abdul-Karim, R. M. 1978. An Introduction to the Taxonomy of the Family Dytiscidae (Coleoptera) of Iraq. A thesis submitted to the College of Science, Bsrah University, $152 \mathrm{pp}$.

Abdul-Karim, R.M. and Ali, H. A. 1986: Description of five new species of Dytiscidae from Iraq. Journal of Biological Science Research, 17: 275-285.

Abdul-Rassoul, M. S. 1976. Checklist of Iraq Natural History Museum Insects collection. Natural. Iraq Natural History Museum Publication, 30: 1- 41. 
Agassi, D. J. L. 2012. The Acentropinae (Lepidoptera: Pyraloidea: Crambidae) of Africa. Zootaxa, 3494: 1-73.

Adawi, S. H, Qasem, K. R., Zawahra, M. M. and Handal, E. N. 2017. On some records of dragonflies (Insecta: Odonata: Anisoptera) from the West Bank (Palestine). Jordan Journal of Biological Sciences, 10(3): 151-157.

Akbar, A., Mehmood, S.A., Panhwar, W.A., Ahmed, S., Tabassum, S., Rajput, S. and Ali, M. 2017. Collection and identification of genus Anax (Odonata, Aeshnidae) from district Swat. Journal of Entomology and Zoology Studies, 5(2): 1440-1442.

Al-Ansari, N., Knutsson, S. and Ali, A. A. 2012. Restoring the Garden of Eden, Iraq. Journal of Earth Sciences and Geotechnical Engineering, 2 (1): 53-88.

Al-Edani, A. A. Z. S. and Kareem D. K. 2015. Diagnosis and ecological distribution of aquatic (Hemiptera: Heteroptera) in Sullein marsh in Basrah, South of Iraq. Mesopotamian Journal of Marine Science, 30 (1): 33 - 46.

Alex, C.J., Soumya, K. C. and V. 2021. A report on the moth (Lepidoptera: Heterocera) diversity of Kavvai River basin in Kerala, India. Journal of Threatened Taxa, 13(2):17753-17779.

Al-Hashmi, A. H., Al-Saffar, H. H. and Augul, R. S. 2018. Key to the species of the Orthetrum Newman, 1833 (Odonata, Libellulidae) with a new record species in Iraq. Bulletin of the Iraq natural. History Museum, 15(1): 15-29.

Alhejoj, I., Sartori, M. and Gattolliat, J. -L. 2020. Contribution to the mayflies (Insecta, Ephemeroptera) of Jordan. Check List, The Journal of Biodiversity data, 16 (2): 237 242.

Al-Hilli, M. R. 1977. Studies on the plant ecology of the Ahwar region in southern Iraq. Ph.D. Thesis, Faculty of Science, University of Cairo, Cairo, Egypt, 357pp.

Al-Houty, W. 2004. A faunistic account of beetles (Coleoptera) of the state of Kuwait. Kuwait Journal of Science and Engineering, 31(1):59-76.

Al-Houty, W. 2009. Insect biodiversity in Kuwait. International Journal of Biodiversity and Conservation, 1(8): 251-257. Available at: http://www.academicjournals.org/ijbc.

Ali, A. H., Azizb, N. M. and Hamza, H. A. 2007. Abundance, occurrence, seasonal changes and species composition of Macroinvertebrates in the restored Iraqi southern marshes. Marsh Bulletin, 2 (1): 80-95. 
Ali, H. A. 1976. Preliminary study on the aquatic beetles of Iraq (Haliplidae, Coleoptera). Bulletin of the Basrah Natural History Museum, 3: 89-94.

Ali, H. A. 1978a. A list of some aquatic beetles of Iraq (Coleoptera: Dytiscidae). Bulletin of the Iraq Natural History Museum, 7(2): 11-14.

Ali, H. A. 1978b. Some taxonomic studies on the aquatic beetles of Iraq (Coleoptera: Gyrinidae). Bulletin of the Iraq Natural History Museum, 7: 15-20.

Ali, W. K. and Khidhir A. Q. S. 2015. Morphological study of the Sympetrum arenicolor Jödicke, 1994 (Odonata: Libellulidae) collected in Kurdistan Region, Iraq. Entomology, Ornithology and Herpetology, 4 (4):168.

Ali, M. H., Anon, M. R. and Mohammed, H. H. 2002. The seasonal variations of abundance and biomass of the two odonate naiads Ischnura evansi Morton (Odonata: Coenagrionidae) and Brachytemis fuscocapillata Selys (Odonata: Libellulideae) in the Qarmat Ali region, Basrah. Marina Mesopotamica, 17: 405-415.

Al-Saffar, M. A. T. 2008. Larval mouthpart deformities in Chironomus annularius Meigen (Diptera: Chironomidae) from Al-Hammar Marsh, Southern Iraq and Tanjero river, Kurdestan, Northern Iraq. Nature Iraq, 12pp.

Amr, Z. S. 2021. The state of biodiversity in Kuwait. Gland, Switzerland: IUCN; the State of Kuwait, Kuwait: Environmental Public Authority, 248pp.

Ananain, V. Yu and Tailly, M. 2013. Additions to the dragonfly (Odonata) fauna of Armenia with new records of rare or uncommon species. Russian Entomological Journal, 22(4): 249-254.

Andersen, N. M. and Weir, T.A. 2004. Mesoveliidae, Hebridae, and Hydrometridae of Australia (Hemiptera:Heteroptera: Gerromorpha), with a reanalysis of the phylogeny of semiaquatic bugs. Invertebrate Systematics, 18: 467-522.

Archer, M. E. 1998. Taxonomy, distribution and nesting biology of Vespa orientalis L. (Hym., Vespidae). Entomologist's Monthly Magazine, 134: 45-51.

Asahina, S. 1973. The Odonata of Iraq. Japanese Journal of Zoology, 17(1): 17-36.

Askew, R. R. 2004. The dragonflies of Europe. Revised edition, Harley Books, Colchester, UK. 308 pp.

Aydin, G. B. and Güher, H. 2017. The Chironomidae (Diptera) fauna of Kirklareli . Province. Turkish Journal of Zoology, 41:335- 341. 


\section{Al-Saffar and Augul}

Aydin, G. B. and Samin, N. 2020. A preliminary study aimed an annotated checklist of Chironomidae (Diptera: Culicomorpha: Chironomoidea) of Iran. Acta Aquatica Turcica, 16(1): 38-50.

Bedair, H. M., Al Saad, H. T. and Salman, N. A. 2006. Iraq's southern marshes something special to be conserved; a case study. Marsh Bulletin, 2(1): 99-126.

Belle, J. 1984. Orthetrum trinacria (Selys) new to the fauna of Spain, with records of three other Afrotropical Odonata Anisoptera. Entomologische Berichten, 44: 79-80.

Betrem, J. G. 1935. Beitrag zur Kenntnis der paläarktischen Arten des genus Scolia. Tijdscherift voor. Entomologie, 78:1-78.

Bippus, M. 2019. Pyraloidea of Mauritius and neighbouring islands (Lepidoptera). Phelsuma, 27: $36-57$.

Boda, P., Bozóki, T., Vásárhelyi, T., Bakonyi, G. and Várbíró, G. 2015. Revised and annotated checklist of aquatic and semi-aquatic Heteroptera of Hungary with comments on biodiversity patterns. ZooKeys, 501: 89-108.

Borisov, S. N. 1987. On the ecology of two similar dragonfly species in Tajikistan. Ekologiya (Moscow), 1: 85-87.

Borkent, A. 1993. A world catalogue of fossil and extant Corethrellidae and Chaoboridae (Diptera), with a listing of references to keys, bionomic information and descriptions of each known life stage. Entomologica Scandinavica, 24: 1-24.

Boudot, J.-P. 2010. Selysiothemis nigra. The IUCN Red List of Threatened Species 2010: e.T165471A6026614. (Accessed on 30 October 2021).

Boudot, J. P. and Kalkman, V. J. (eds.) 2015. Atlas of the European Dragonflies and Damselflies. KNNV Publishing, The Netherlands, 381 pp.

Boudot, J. P. and Salamun, A. 2015. Ischnura elegans (Vander Linden, 1820). In: Boudot, J. P. and Kalkman, V. J. (eds.), Atlas of the European Dragonflies and Damselflies. KNNV Publishing, The Netherlands, 381 pp.

Boudot, J-P., Kalkman, V. J., Amorín, M.A., Bogdanović, T., Rivera, A. C., Degbriele, G., Dommanget, J-L., Ferreira, S., Garrigós, B., Jović, M., Kotarac, M. Lopau, W., Marinov, M., Mihoković, N., Riservato, E., Samraoui, B. and Schneider, W. 2009. Atlas of the Odonata of the Mediterranean and North Africa. Libellula Supplement, 9: $1-256$. 
Boudot, J.-P., Clausnitzer, V., Samraoui, B., Suhling, F., Dijkstra, K.-D. B. and Schneider, W. 2016. Orthetrum chrysostigma. The IUCN Red List of Threatened Species 2016: e.T59954A83856823. https://dx.doi.org/10.2305/IUCN.UK.20163.RLTS.T59954A83856823.en. (Accessed on 30 October 2021).

Brinck, P. 1955. A revision of the Gyrinidae (Coleoptera) of the Ethiopian region I. Lunds Universitets Årsskrift (Neue Folge) (Avd. 2) 51 (16). Kungliga Fysiografi ska Sällskapets Handlingar (Neue Folge), 66(16): 1-141.

Cannings, S. G. and Cannings, R. A. 1985. The larva of Somatochlora sahlbergi Trybŏm, with notes on the species in the Yukon Territory, Canada (Anisoptera: Corduliidae). Odonatologica, 14 (4): 319-330.

Carpenter, J. M. 1986. A synonymic generic checklist of the Eumeninae (Hymenoptera: Vespidae). Psyche, 93: 61-90.

Carpenter, J. M. 1996. Distributional checklist of species of the genus Polistes (Hymenoptera: Vespidae; Polistinae, Polistini). American Museum Novitates, 3188: 1-39.

Castro, L. and Dvořák, L. 2010. New and noteworthy records of vespid wasps (Hymenoptera: Vespidae) from the Palaearctic region (III). Acta Musei Moraviae, Scientiae Biologicae, 95(2): 37-53.

Catalogue of Life, 2009. Annual checklist. Available at: http://www.catalogueoflife.org/annualchecklist/2009/search.php

Chahartaghi Abineh, M. 2002. Systematical study on the Scoliid fauna (Hym.: Aculeata) in Karaj vicinity (Tehran province).Thesis of M.Sc. Agricultural Entomology, Karaj, Iran, 128 pp.

Chaplina, I. A. 2004. Fauna and ecology of Odonata of Kazakhstan. Autoreferat of Ph. D. Thesis of biol. speciality, Novosibirsk: 23. (In Russian).

Chen, F., Song, S. and Wu, C. 2006. A review of the genus Parapoynx Hübner in China (Lepidoptera: Pyralidae: Acentropinae). Aquatic Insects, 28(4): 291- 303.

Clausnitzer, V. 2013. Sympetrum fonscolombii. The IUCN Red List of Threatened Species 2013: e.T60038A17538409. https://dx.doi.org/10.2305/IUCN.UK.20131.RLTS.T60038A17538409.en. (Accessed on 30 October 2021).

Clausnitzer, V. 2020. Sympetrum striolatum. The IUCN Red List of Threatened Species 2020: e.T158685A83884411. https://dx.doi.org/10.2305/IUCN.UK.20203.RLTS.T158685A83884411.en. (Accessed on 30 October 2021) 
Al-Saffar and Augul

Clausnitzer, V., Dijkstra, K. -D. B., Koch, R. Boudot, J. -P., Darwall, W. R. T. Kipping, J., Samraoui, B., Samways, M. J., Simaika, J. P. and Suhling, F. 2012. Focus on African Freshwaters: hotspots of dragonfly diversity and conservation concern. Frontiers in Ecology and the Environment, 10: 129-134.

Cranston, P. S. and Judd, D. D. 1989. Diptera: Fam. Chirononlidae of the Arabian Peninsula. Fauna of Saudi Arabia, 10: 236-289.

Coe, R. L., Freeman, P. and Mattingly, P. F. 1950. Diptera 2.Nematocera: families Tipulidae to Chiroomidae, Chironomidae, Handbook for the identification of British Iinsects, Royal of the Entomological Societyof London, 9(2):121-216.

Corbet, P. S.1999. Dragonflies: behaviour and ecology of Odonata. Aquatic Insects, 23(1):83.

Darilmaz, C. and Kiyak, S. 2009. Checklist of Gyrinidae, Haliplidae, Noteridae and Dytiscidae of Turkey (Coleoptera: Adephaga). Journal of Natural History, 43(15-26): 25-26.

Das, B. P., Gupta, V. K. 1989. The social wasps of India and the adjacent countries (Hymenoptera: Vespidae). Oriental Insects. Monograph 11. Gainesville, Florida, USA: Association for the Study of Oriental Insects, $292 \mathrm{pp}$.

De Knijf, G. and Demolder, H. 2013. Early spring observations of Odonata from Cyprus. Libellula, 32 (1/2): 59-74.

De Prins, J. and De Prins, W. 2019. Afromoths, online database of Afrotropical moth species (Lepidoptera). World Wide Web electronic publication (www.afromoths.net) (Accessed on 01.ix.2019).

Derwesh, A. I. 1965. A preliminary list of identified insects and arachnids of Iraq. Director General Agriculture Research Projections Baghdad, Bulletin, 112:121-123.

Dijkstra, K-D. B and Lewington, R. 2006. Field Guide to the Dragonflies of Britain and Europe. British Wildlife Publishing, 320 pp.

Dubatolov, V. V. 2019. Vespidae collection of Siberian Zoological Museum. Available at: http://szmn.eco.nsc.ru/Hymenop/Vespidae.htm.

Dumont, H. J. 1991. Fauna palaestina. Insecta V - Odonata of the Levant. Israel Academy of Sciences and Humanities, Jerusalem, 297 pp.

Edmonds, N. J., Foster, G. N., Davison, P. I. and Al-Zaidan, A. S. 2019. Additional records of aquatic Coleoptera from Kuwait (Coleoptera: Noteridae, Dytiscidae, Spercheidae, Hydrophilidae). Koleopterologische Rundschau, 89:11-15. 
Egan, A. T., JR Ferrington, L. C. 2019. Chironomidae of the Upper Saint Croix River, Wisconsin. Transactions of the American Entomological Society, 145(3):353- 384.

Enkhnasan, D. and Blodgiv, B. 2019. Biogeography of predaceous diving beetles (Coleoptera, Dytiscidae) of Mongolia. Zookeys, 853:87-108.

Fallahzadeh, M. and Saghaei, N. 2010. A brief study on the Scoliidae (Insecta: Hymenoptera) in Iran. Munis Entomology and Zoology, 5(2):792-795.

Forster, V. W. 1963. Ergebnisse der Zoologischen Nubien-Expedition 1962, Teil XI, Lepidoptera: Rhopalocera. Annalen des Naturhistorischen Museums in Wien, 66:457-458.

Garrido, J. and Munilla, I. 2008. Aquatic Coleoptera and Hemiptera assemblages in three coastal lagoons of the NW Iberian Peninsula: assessment of conservation value and response to environmental factors. Aquatic Conservation: Marine and Freshwater Ecosystems, 18: 557-569.

Gashtarov, V. and Beshkov, S. 2010. Lindenia tetraphylla (Vander Linden, 1825) (Odonata: Gomphidae) a new genus and species for the Bulgarian fauna. The Entomologist's Record and Journal of Variation, 122: 272-274.

GBIF Secretariat. 2021. GBIF Backbone Taxonomy. Checklist dataset https://doi.org/10.15468/39omei accessed via GBIF.org on 2021-10-18.

Geraci, C. J., Zhou, X. and Al-Saffar, M. 2011. Barcoding Iraq: Aquatic Insects of the Tigris/Euphrates River Basin Useful for Biosurveillance. $4^{\text {th }}$ International Barcode of Life Conference, Adelaide, Australia.

Ghahari, H. 2013. A study on aquatic and semiaquatic bugs (Hemiptera: Heteroptera) from northern Iran. Linzer biologische Beiträge, 45(2): 1991-1996.

Grandova, M. A. 2013. Aquatic Heteroptera (Nepomorpha, Gerromorpha) in small intermittent rivers of Ukraine steppe zone. ZooKeys, 319: 107-118.

Guershon, M. and Ionescu-Hirsch, A. 2012. A review of the Xylocopa species (Hymenoptera: Apidae) of Israel. Israel Journal of Entomology, 41-42:145-163.

Guignot, F. 1961. Revision des hydrocanthares d'Afrique (Coleoptera Dytiscoidea), troisième partie. Annales du Musée Royal du Congo Belge, Série 8vo Sciences Zoologiques), 90: 659-995. 


\section{Al-Saffar and Augul}

Hacet, N. 2017. Updated checklist of Odonata fauna in the Turkish Thrace Region, with additional records of new, rare, and threatened taxa. Turkish Journal of Zoology, 41(2): 33-42.

Hacet, N. and Aktaç, N. 1997. Istranka Dağlari Odonata Faunasi. Turkish Journal of zoology, 21: 275-289.

Hájek,J. 2006. The westernmost record of Neptosternus circumductus, and a review of Dytiscidae (Coleoptera) of Baluchistan (Iran, Pakistan). Acta Entomologica Musei Nationalis Pragae, 46: 43-56.

Harbach, R. E. and Kitching, I. 2016. The phylogeny of Anophelinae revisited: inferences about the origin and classification of Anopheles (Diptera: Culicidae). Zoologica Scripta, 45: 34-47.

Hanson, B. A., Mushet, D., Euliss, N. H., Chordas, S. 2007. First Record of Corisella inscripta (Uhler) (Heteroptera: Corixidae) from North Dakota. Prairie Naturalist, 39 (2):107-110.

Hassan, K. S., Habeeb, M. A. and Al-Mousawi, N. J. 2000. Occurence of aquatic insects with algae in Basrah Province. Marina Mesopotamica, 15: 137-143.

Heidari, H. and Dumont, H. J. 2002. An annotated check-list of the Odonata of Iran. Zoology in the Middle East, 2 6:1 33-150.

Henry, T. J. and Froeschner, R. C.(eds) 1988. Catalog of the Heteroptera, or True Bugs of Canada and the Continental United States. Brill Academic Publishers, 958 pp.

Hermans, J. and Gubbels, R. 1997. De vuurlibel (Crocothemis erythraea (Brullé)) in Limburg. Brachytron, 1 (1): 22-26.

Hussain, N. A. 2014. Biotopes of the Iraqi marshes, First Edition. Dhifaf publishing house, Basra, Iraq, 432 pp.

Insectomania. 2020. Heteroptera Miridae: Systematic Part. Available at: https://www.insectomania.org/heteroptera-miridae/systematic-part.html. (Accessed on 20 October 2021)

Iraqi Ministry of Health and Environment. 2017. Biodiversity survey report in the marshes of Southern Iraq, $61 \quad$ pp. Available at: https://moen.gov.iq/Portals/11/\%D8\%A7\%D9\%84\%D9\%85\%D8\%B9\%D8\%A7\%D8 $\%$ B1\%D9\%81/Biodiversity\%20Survey\%20Report.pdf 
Jaczewski, T. 1964. Notes on some aquatic and semiaquatic Heteroptera from Iraq. Bulletin d'Académie Polonaise des Sciences Cl. 2. Série des Sciences Biologiques, 12(6): 263268 .

Jödicke, R. 2003. Mid-winter occurrence of dragonflies in southern Tunisia (Insecta: Odonata). Kaupia Darmstaedter Beitraege zur Naturgeschichte, 12: 119-128.

Jones, S. 1996. First British record of the scarlet dragonfly Crocothemis erythraea (Brullé). Journal of the British Dragonfly Society, 12 (1): 11-12.

Jung, S. W., Min, H. K. and Lee, D. -H. 2020. Aquatic Beetles Fauna in Nohwa and Bogil Islands, and Copelatus parallelus (Coleoptera: Dytiscidae) and Scirtes sobrinus (Coleoptera: Scirtidae) New to South Korea. Animal Systymatic, Evolution and Diversity, 36(2): 128-138.

Kalkman, V. J. 2006a. Key to the dragonflies of Turkey. Brachytron, 10 (1): 3-82.

Kalkman, V. J. 2006 b. Key to the dragonflies of Turkey including species known from Greece, Bulgaria, Lebanon, Syria, the Trans-Caucasus and Iran. Brachytron, 10 (1): 382.

Kalkman, V. J. and Wasscher, M. 2003. An annotated checklist of the Odonata of Turkey. Odonatologica, 32 (3): 215-236.

Katbeh-Bader, A., Amr, Z., Baker, M. A. and Mahasneh, A. 2004. The dragonflies (Insecta: Odonata) of Jordan. Denisia, 14:309- 317.

Kazenas, V. L. 2014. Collection materials on eusocial wasps (Hymenoptera, Vespidae: Vespinae et Polystinae) of Kazakhstan in the Institute of Zoology of the Ministry of Education and Science of the Republic of Kazakhstan (Almaty). Selevinia, 22: 193-196 (in Russian).

Kerr, W. E. 1957. Introdução de abelhas africanas no Brasil. Brasil Apicola, 3:211-213.

Khalaf, K. T. 1958. Some Hymenoptera and Coleoptera from Iraq. Iraq Natural History Museum Publication, 14: 1-3.

Kimmins, D. E. 1960. The Ephemeroptera types of species described by A. E. Eaton, R. McLachlan and F. Walker, with particular reference to those in the British Museum (Natural History). Bulletin of the British Museum (Natural History) Entomology, 9:269-318. 
Al-Saffar and Augul

Knijf, G. 2003. Verslag van de excursie van 15 juni 2003 naar de Limburgse Hoge Kempen (Ruwmortelsven - Kruisven -Vallei van de Asbeek - Vallei van de Zijpbeek). Gomphus, 19 (2): 90-92.

Kunz, B. and Kunz, D. 2001. Lindenia tetraphylla Wiederfund für Nordafrika (Odonata: Gomphidae).Libellula, 20: 79-85.

Kumar, P. G. 2012. Redescription and new distributional records of Delta Esuriens (Fabricius) (Hymenoptera: Vespidae: Eumeninae) from Indian States. Records of the Zoological Survey of India, 112(Part-4): 55-60.

Larocque-Tobler, I. 2014. The Polish sub-fossil chironomids. Palaeontologia Electronica, 17 (1,3A): 1- 28 .

Lauck, D. R. and Menke, A. 1961. The higher classification of the Belostomatidae (Hemiptera). Annals of the Entomological Society of America, 54: 644-657.

Linnavuori, R. E. 1994. Hemiptera of Iraq. IV. Heteroptera, the aquatic and subaquatic families, Saldidae and Leptopodidae. Entomologica Fennica, 5:87-95.

Lőkkös, A. 2014. The water and shore beetles (Coleoptera) of the Kis-Balaton. Natura Somogyiensis, 25: 141-156.

Madden, C. P. 2010. Key to genera of larvae of Australian Chironomidae (Diptera). Museum Victoria Science Reports, 12: 1-31.

Madl, M. 1997. Über Vespiden, Pompiliden, Scoliiden und Tiphiiden der Türkie (Hymenoptera). Linzer biologische Beiträge, 29 (2): 823-827.

Malikova, E. 2009. Sympetrum arenicolor. The IUCN Red List of Threatened Species 2009: e.T158718A5272818. https://dx.doi.org/10.2305/IUCN.UK.20092.RLTS.T158718A5272818.en. (Accessed on 30 October 2021).

Mazzoldi, P. 2015. World checklist of freshwater Coleoptera: Gyrinidae species. World Wide Web electronic publication. Available at: http://fada.biodiversity.be/group/show/63

Menetrey, N., Oertli, B., Sartori, M., Wagnerm A. and Lachavanne, J. B. 2008. Eutrophication: are mayflies (Ephemeroptera) good bioindicators for ponds? Hydrobiologia, 597 (1): 125-135.

Miller, K. B. 2002. Revision of the genus Eretes Laporte, 1833 (Coleoptera: Dytiscidae). Aquatic Insects, 24(4): 247-272. 
Mitra, A. 2013. Orthetrum taeniolatum. The IUCN Red List of Threatened Species 2013: e.T165506A17533964. https://dx.doi.org/10.2305/IUCN.UK.20131.RLTS.T165506A17533964.en. (Accessed on 30 October 2021).

Mitra, A. 2016. Anax imperator. The IUCN Red List of Threatened Species 2016: e.T59812A72311295. Available at: https://dx.doi.org/10.2305/IUCN.UK.20163.RLTS.T59812A72311295.en. (Accessed on 29 October 2021).

Mitra, A. and Clausnitzer, V. 2018. Anax parthenope. The IUCN Red List of Threatened Species 2018: e.T165488A72312377. https://dx.doi.org/10.2305/IUCN.UK.2018 1.RLTS.T165488A72312377 .en. (Accessed on 29 October 2021).

Morice, F. D. 1921. Annotated lists of aculeate Hymenoptera (except Heterogyna) and chrysids recently collected in Mesopotamia and north-west Persia. Journal of the Bombay Natural History Society, 28: 192-203.

Moritz, R. F. A., Hartel, S. and Neumann, P. 2005. Global invasions of the western honeybee (Apis mellifera) and the consequences for biodiversity. Ecoscience, 12(3):289-301.

Morton, K. J. 1919. Odonata from Mesopotamia. Entomologist's Monthly Magazine, 55: 143151.

Morton, K. J. 1920. "Odonata collected in north-western Persia and Mesopotamia by Captain P. X. Buxton - R. A. M. C. Entomologist's Monthly Magazine, 56: 82-87.

Morton, K. J. 1924. The Dragonflies (Odonata) of Palestine based primarily on collection made by Dr. P.A. Buxton, with notes on the species of the adjscent regions. Transactions of the Entomological Society of London, 29:25-44.

Muller, K.1987. Die Pokalazurjungfer (Cercion lindeni Navas) und die Feuerlibelle (Crocothemis erythraea Brulle) im mittleren Remstal. Libellula, 6 (3-4): 82-83.

Müller-Liebenau, I. and Hubbard, M. D. 1985. Baetidae from Sri Lanka with some general remarks on the Baetidae of the oriental region (Insecta: Ephemerptera). Florida Entomology, 68:537-561.

Mohammadi, H., Ghaderi, E., Ghorbani, F., Mansouri, A. and Namayandeh, A. 2021. Chironomidae (Diptera: Insecta) from Sirwan River watershed of Kurdistan (Iran) with new faunistic records for Iran and range extensions for the Palearctic region. Biologia, 76: 1227-1253.

Na, K. B., Ree, H. I., Jung, S. W. and Bae, Y. J. 2010. Chironomidae (Diptera) fauna of Seoul-Gyeonggi Area in Korea. Entomological Research Bulletin, 26: 59-67. 
Nelson, B., Cummins, S., Fay,L., Jeffrey,R., Kelly, S., Kingston, N., Lockhart, N., Marnell, F., Tierney, D. and Jackson, W.2019 Checklists of protected and threatened species in Ireland. National Parks and Wildlife Service, Irish Wildlife Manuals, 116: 1-48.

Nguyen, L. T. P., Khuat, L. D., Do, L. V. and Luong, H. V. 2007. Survey of bees and wasps (Hymenoptera) in Cuc Phuong National Park Proceedings of the 2nd National Scientific Conference on Ecology and Biological Resources, Hanoi 26 Oct 2007 (in Vietnamese), p 415 - 419.

Nilsson, A. N. 2006. Which name is valid - Hygrobiidae or Paelobiidae? Latissimus, 21: 37 39.

Nilsson, A. N. 2011. A world catalogue of the family Noteridae, or the burrowing water beetles (Coleoptera, Adephaga). Version 16.VIII.2011, Available at: http://www.waterbeetles.eu/documents/W_CAT_Noteridae.pdf.

Nilsson, A. N. and Hájek, J. 2021. A world catalogue of the family Dytiscidae, or the diving beetles (Coleoptera, Adephaga). Version 1.I.2021, 315 pp. Available at: http://www.waterbeetles.eu/ Accessed 1 Jul 2021.

Nilsson, A. N., Wewalka, G., Wang, L. -L. and Sato, M. 1995. An annotated list of Dytiscidae (Coleóptera) recorded from Taiwan. Beitraege-zur-Entomologie, 45(2): 357-374.

Nieser, N. 2004. Guide to aquatic Heteroptera oF Singapore and Peninsular Malaysia III. Pleidae and Notonectidae. The Raffles Bulletin of Zoology, 52(1): 79-96.

Novoselsky, T., Chen, P. P. and Nieser, N. 2018. A review of the giant water bugs (Hemiptera: Heteroptera: Nepomorpha: Belostomatidae) of Israel. Israel Journal of Entomology, 48 (1): 119-141.

Oliver, D. R., Dillo, M. E. and Cranst, P. S. 1990. A catalogue of Nearctic Chironomidae. 98pp.

Ornosa, C., Ortiz-Sanchez, F. J. and Torres, F. 2007. Catalogo de los Megachilidae del Mediterraneo occidental (Hymenoptera, Apoidea). II. Lithurgini y Megachilini. Graellsia, 63(1):113-134.

Osten, T. 2000. Die Scoliiden des Mittelmeer-Gebietes und angrenzender Regionen (Hymenoptera) Ein Bestimmungsschlüssel. Linzer biologische Beiträge, 32(2):537593.

Osten, T. 2005a. Beitrag zur Kenntnis der Scoliiden fauna des Oman (Hymenoptera, Scoliidae). Entomofauna, 26(2): 9-16. 
Osten, T. 2005b. Die Scoliiden-Fauna Mittelasiens (Kasakhstan, Turkmenistan, Uzbekistan, Tadzikistan, Kirgistan) Ein Bestimmungsschlüssel. Linzer biologische Beiträge, 37 (2):1451-1479.

Osten, T. and Arens, W. 2004. Beitrag zur Kenntnis der Scoliiden-Fauna Griechenlands (ohne Zypern) (Hymenoptera, Scoliidae). Entomofauna, 25 (20): 305-320.

Osten, T., Ebrahimi, E. and Chahartaghi, A. M. 2003. Die Scoliiden des Iran und angrenzender Regionen mit Anmerkungen $\mathrm{zu}$ ihrer Lebensweise (Hymenoptera, Scoliidae). Entomofauna, 24 (26):353-377.

Perez-Goodwyn, P. J. 2006. Taxonomic revision of the subfamily Lethocerinae Lauck \& Menke (Heteroptera: Belostomatidae). Stuttgarter Beiträge zur Naturkunde, A (Biologie), 695: 1-71.

Perveen, F., Khan, A. and Abdul Rauf, S. 2014. Key for first recorded dragonfly (Odonata: Anisoptera) fauna of district Lower Dir, Khyber Pakhtunkhwa, Pakistan. International Research Journal of Insect Sciences, 1(2): 26-35.

Perissinotto, R., Bird, M. and Bilton, D. T. 2016. Predaceous water beetles (Coleoptera, Hydradephaga) of the Lake St Lucia system, South Africa: biodiversity, community ecology and conservation implications. ZooKeys, 595: 85-135.

Ramadan, M. M. and Ramadan, H. M. 2021. Phenology of predaceous diving beetles (Coleoptera: Dytiscidae) in a Desert Oasis. International Journal of Zoology and Animal Biology, 4 (2): 000280.

Reels, G. T. 2010. Seasonal emergence of dragonflies (Odonata: Anisoptera) at ten ponds in Hong Kong. Hong Kong Entomological Society, 2(1): 24-31.

Rehfeldt, G. 1991. Site-specific matefinding strategies and oviposition behavior in Crocothemis erythraea (Brulle) (Odonata: Libellulidae). Journal of Insect Behavior, 4 (3): 293-303.

Richardson, C. J., Reiss, P., Hussain, N. A., Alwash, A. J. and Pool, D. J. 2005. The restoration potential of the Mesopotamian marshes of Iraq. Science, 307: 1307-1311.

Rossaro, B., Pirola, N., Marziali, L., Magoga, G., Angela, B. and Montagna, M. 2019. An updated list of chironomid species from Italy with biogeographic considerations (Diptera, Chironomidae). Biogeographia - The Journal of Integrative Biogeography, 34:59-85.

Ruttner, F. 2003. Biogeography and taxonomy of honeybees. Springer, Berlin, 1988, xii+284 pp. 
Al-Saffar and Augul

Sadeghi, S., Kyndt, T. and Dumont, H. J. 2010. Genetic diversity, population structure and taxonomy of Calopteryx splendens (Odonata: Calopterygidae): an AFLP analysis. European Journal of Entomology, 107 (2): 137-146.

Salah, M. and Cueto, J.A.R. An annotated checklist of the aquatic Adephaga (Coleoptera) of Egypt. II. Dytiscidae: Hydroporinae. Boletín de la Sociedad Entomológica Aragonesa (S.E.A.), no 54 (30/6/2014): 293-305.

Salur, A. and Özsaraç, Ö. 2004. Additional notes on the Odonata fauna of Çiçekdaği (Kirşehir), Turkey. Gazi University Journal of Science, 17(1):11-19.

Sasa, M. 1989. Chironomidae of japan: Checklist of species recorded, key to males and taxonomic notes. Research Report from the National Institute for EnvironmentalStudies, Japan, No. 125, 189pp.

Schneider, T., Kemeyer, D., Müller, O. and Dumont, H. J. 2018. Checklist of the dragonflies (Odonata) of Iran with new records and notes on distribution and taxonomy. Zootaxa, 4394 (1): 1-40.

Schneider, W. 1985. Die Gattung Crocothemis Brauer 1868 im Nahen Osten (Insecta: Odonata: Libellulidae). Senckenbergiana Biologica, 66 (1-3): 79-88.

Schneider, W. 1995. Eine Paarungskette zwischen Orthetrum sabina (Drury, 1770) und Crocothemis erythraea (Brulle 1832) (Odonata: Anisoptera: Libellulidae). Entomologische Zeitschrift, 105 (23): 462-463.

Schröter, A. 2010. On a collection of dragonflies from eastern Georgia, with the first record of Sympetrum arenicolor (Odonata: Libellulidae). Libellula, 29 (3/4): 209-222.

Schroter, A., Seehausen, M., Kunz, B., Gunther, A., Schneider, T., and Jodicke, R. 2015. Update of the Odonata fauna of Georgia, southern Caucasus ecoregion. Odonatologica, 44(3), 279-342.

Schweiger, L. 1983. Zum Vorkommen und Status der Feuerlibelle (Crocothemis erythraea Brulle, 1832) in Österreich (Insecta: Odonata, Libellulidae). Berichte des Naturwissenschaftlich Medizinischen Vereins in Innsbruck, 70: 105-110.

Shalaby, F. A. 1961. Preliminary survey of the insect fauna of Saudi Arabia. Bulletin de la Société Entomologique d'Egypte, 45:211-225.

Sharma, G. and Clausnitzer, V. 2016. Ischnura senegalensis. The IUCN Red List of Threatened Species 2016: e.T59897A75436136. https://dx.doi.org/10.2305/IUCN.UK.2016-3.RLTS.T59897A75436136.en.

(Accessed on 29 October 2021). 
Sheppard, W. S. and Meixner, M. D. 2003. Apis mellifera pomonella, a new Honeybee subspecies from Central Asia. Apidologie, 34(4):367-175.

Schuh, R. T. and Weirauch, C. 2020. True bugs of the world (Hemiptera: Heteroptera): classification and natural history. $2^{\text {nd }}$ Edition. Siri Scientific Press, Manchester, 767 pp.

Scott, D. A. (ed.) 1995. A directory of wetlands in the Middle East. IUCN, Gland, Switzerland and IWRB, Slimbridge, U.K. xvii +560 pp.

Speidel, W. and Mey, W. 1999. Catalogue of the oriental Acentropinae (Lepidoptera, Crambidae). Tijdschrift voor Entomologie ,142: 125-142. Downloaded from Brill.com10/25/2021 05:18:20AM. via free access.

Srinivasan, G. and Kumar, P. G. 2010. New records of potter wasps (Hymenoptera: Vespidae: Eumeninae) from Arunachal Pradesh, India: five genera and ten species. Journal of Threatened Taxa, 2(12):1313-1322.

Steinberg, A. M. 1962. Gen. Scolia (Scoliidae). Fauna der USSR, 13: 1-185.

Steinmann, H. 1997. World catalogue of Odonata II. Anisoptera. Das Tierreich/The Animal Kingdom. Wermuth, H. and Fischer, M., (eds.); Teilband/Part 111., W. de Gruyter, Berlin-New York, 636 pp.

Subramanian, K. A., Emiliyamma, K. G., Babu, R., Radhakrishnan, C. and Talmale, S. S. 2018. Atlas of Odonata (Insecta) of the Western Ghats, p. 1-417. Zoological Survey of India, Kolkata.

Taher, M. and Heydarnejad, M.S. 2019. Aquatic Beetles (Coleoptera: Dytiscidae, Haliplidae, Noteridae, Hydrophilidae) From Borujen and Lordegan (Chaharmahal and Bakhtiari Province, Iran). Journal of Zoological Research 1(2): 13-16.

Terzani, F. 2002. Ricerche odonatologiche in Toscana 8. La Lindenia tetraphylla (Vander Linden, 1825). Quaderno di Studi e Notizie di Storia naturale della Romagna, 16: 5-6.

Trilar, T. and Bedjanič, M. 1999. Contribution to the knowledge of the dragonfly fauna of Lastovo Island, Dalmatia, southern Croatia. Exuviae, 6: 1-6.

Tungpairojwong, N., Nguyen, V. V. and Bae, Y.J, 2006. Procloeon spi-nosum, a new species of Baetidae (Insecta: Ephemeropetra) from Vietnam. Korean Journal of Systematic Zoology, 22:145-147.

Turić, N., Merdić, E., Kutuzović, B. H., Jeličić, Ž. and Bogdanović, T. 2011. Diversity of aquatic insects (Heteroptera: Nepomorpha, Gerromorpha and Coleoptera: 


\section{Al-Saffar and Augul}

Hydradephaga, Hydrophilidae) in the karst area of Gorski kotar, Croatia. Natura Croatica, 20(1):179-188.

Turić, N., Temunović, M., Vignjević, G., Antmunović dunić, J. and Merdić, E. 2017. A comparison of methods for sampling aquatic insects (Heteroptera and Coleoptera) of different body sizes, in different habitats using different baits. European Journal of Entomology, 114:123-132.

Vafaei, R., Darilmaz, M.C., Nazari, E., Incekara, U. and Piazak, N. 2009. Contributions to the knowledge of Iranian aquatic Coleoptera fauna (Dytiscidae, Gyrinidae, Helophoridae and Hydrophilidae) with new records and notes on the rare species Coleostoma transcaspicum Reitter, 1906. Acta entomologica serbica, 14(1): 101-107.

Villastrigo, A., Jäch, M. A., Cardoso, A., Valladares, L. F. and Ribera, I. 2019. A molecular phylogeny of the tribe Ochthebiini (Coleoptera, Hydraenidae, Ochthebiinae). Systematic Entomology, 44: 273-288.

Wagner, R., Barták, M., Borkent, A., Courtney, G., Goddeeris, B., Haenni, J.-P., Knutson, L., Pont, A., Rotheray, G. E., Rozkošný, R., Sinclair, B., Woodley, N., Zatwarnicki, T.and Zwick P.. 2008. Global diversity of dipteran families (Insecta Diptera) in freshwater (excluding Simulidae, Culicidae, Chironomidae, Tipulidae and Tabanidae). Hydrobiologia, 595: 489-519.

Waltz, R. D. and McCafferty, W. P. 1985. A new species of Procloeon from Taiwan (Ephemeroptera: Baetidae). Oriental Insects, 19:121-123.

Wancke, K. 1982. Die Hozbienen des vorderen Orients (Hymenoptera: Apidae). Linzer Biologie Beitrage, 14(1): 23-37.

Yapo, L. M., Célestin B., Atsé, C. B. and Kouassi, P. 2013. Composition, abundance and diversity of aquatic insects in fishponds of southern Ivory Coast, West Africa. Entomologie Faunistique - Faunistic Entomology , 66: 123-133.

Yen, C. C. and Dawood, M. M. 2021. Dragonflies and Damselflies (Odonata) of Kadamaian, Kinabalu Park, Sabah. Journal of Tropical Biology and Conservation, 18: 71-79.

Yıldırım, E. and Kojima, J.1999. Distributional checklist of the species of the family Vespidae (Insecta: Hymenoptera; Aculeata) of Turkey. Natural History Bulletin of Ibaraki University, 3: 19-5.

Younes, A. A. 2008. Predation of the Diving Beetle, Eretes sticticus (Coleoptera: Dytiscidae) on Mosquito Larvae, Culex pipiens L. (Diptera: Culicidae). Egyptian Journal of Biological Pest Control, 18(2): 303-308. 


\section{Survey of insects}

Young, F. N. 1985. A key to the American species of Hydrocanthus Say, with descriptions of new taxa (Coleoptera: Noteridae). Proceedings of the Academy of Natural Sciences of Philadelphia, 137: 90-98.

Zazanashvili, N. and Mallon, D. (eds.) 2009. Status and Protection of Globally Threatened Species in the Caucasus. Tbilisi: CEPF, WWF. Contour Ltd., 232 pp.

Zettel, H. 2014. Annotated catalogue of the semi-aquatic bugs (Hemiptera: Heteroptera: Gerromorpha) of Luzon Island, the Philippines, with descriptions of new species. Zeitschrift der Arbeitsgemeinschaft Österreichischer Entomologen, 66: 85-140.

Zhang, H. -M., Vogt, T. E. and Cai, Q.-H. 2014. Somatochlora shennong sp. nov. from Hubei, China (Odonata: Corduliidae). Zootaxa, 3878 (5):479-84. 
Al-Saffar and Augul

Bull. Iraq nat. Hist. Mus.

(2021) 16 (4): 571-621.

$$
\begin{aligned}
& \text { مسح للحشرات في بعض اهوار جنوب العراق } \\
& \text { هناء هاني الصفار و رزاق شعلان عكل } \\
& \text { مركز بحوث و متحف التاريخ الطبيعي، جامعة بغداد، بغداد، العراق. } \\
& \text { تأريخ الاستلام: 2021/09/13، تأريخ القبول: 2021/11/28، تأريخ النشر: 2021/12/20 } \\
& \text { الخلاصة } \\
& \text { تضمنت التحريات الحالية مسح و مراجعة للاسماء العلمية لحشرات الاهوار (المائية } \\
& \text { وما حولها) لتكون قاعدة بيانات موحدة و محدثاة. }
\end{aligned}
$$

اظهر المسح 109 نوعا تعود الى 77 جنسا ضمن 32 عائلة و 7 رتب و كما ياتي :رتبة غمدية الاجنحة: 44 نوعا ، رتبة ثنائية الاجنحاة:7 انواع، رتبة ذبابة مايو: نوعين ، و رتبة نصفية الاجنحة: 14 نوعا، رتبة غشـائية الاجنحة: 11 نوعا ، حرشفية الاجنحة: نوعين،

$$
\text { و رتبة الرعاشات: } 29 \text { نوعا. }
$$

تضمن التحري مراجعة الاسماء العلمية الشرعية و مرادفاتها و توزيعها الجغرافي العالمي و مكان و تاريخ الجمع للعينات المدروسـة. 